Aus dem Institut/der Klinik für Zahn-, Mund- und Kieferheilkunde der Medizinischen Fakultät Charité - Universitätsmedizin Berlin

\author{
DISSERTATION
}

Changing Dentists' Carious Tissue Removal Behavior: Qualitative Study and Behavioral Change Simulation Experiment

\author{
zur Erlangung des akademischen Grades \\ Doctor medicinae dentariae (Dr. med. dent.) \\ vorgelegt der Medizinischen Fakultät \\ Charité - Universitätsmedizin Berlin
}

von

Linda-Maria Jeggle-Engbert

aus Ahlen (Westf.)

Datum der Promotion: 13. Dezember 2019 


\section{Widmung}

Für meinen Vater Christoph und meinen Opa Matthias 


\section{Inhaltsverzeichnis}

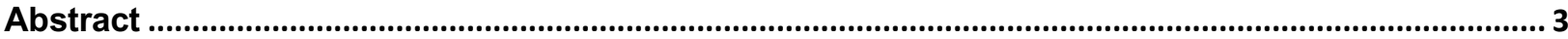

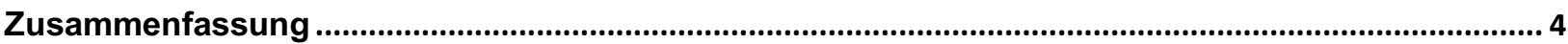

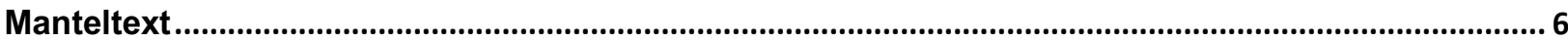

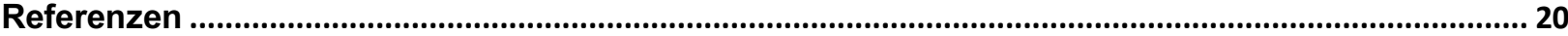

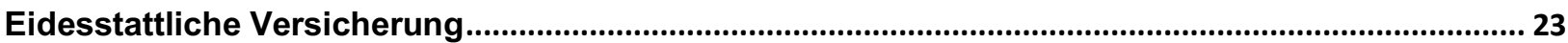

Anteilserklärung an der ausgewähIten Publikation .................................................................................... 24

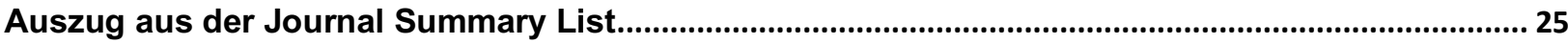

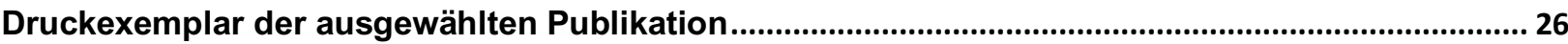

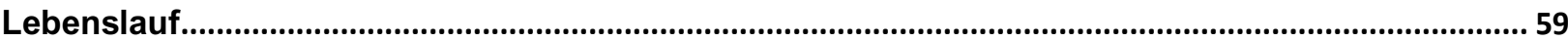

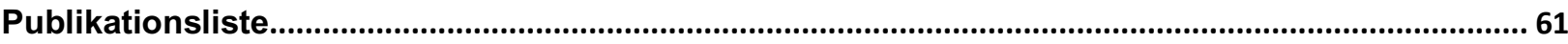

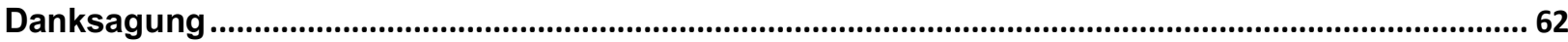




\title{
Changing Dentists' Carious Tissue Removal Behavior: Qualitative Study and Behavioral Change Simulation Experiment
}

\begin{abstract}
Objectives: We aimed to understand why German dentists remain reluctant about selective carious tissue removal (SE), and to develop and test two interventions for changing dentists' behavior.
\end{abstract}

Methods: Ten one-to-one interviews with German dentists were conducted, and identified themes linked to the Behavioral Change Wheel to develop two interventions. The intervention "Guideline" summarized a scientific statement on SE, while the intervention "Tool" simulated dentists having a removal tool (self-limiting handpiece) allowing them to reliably perform SE. For testing these interventions, a postal behavioral-change simulation- experiment was performed on German dentists ( $n=1226$ / intervention), delivered via sealed envelopes. Dentists were first, without knowledge of the intervention, asked to fill out a questionnaire, including a question on their simulated removal behavior in deep lesions in vital teeth, measured via the dentin hardness dentists would leave close to the pulp. After opening the sealed envelope and receiving the simulated intervention, dentists filled out a second identical questionnaire.

Results: Based on identified barriers (lack of guidelines, discrepancy between established and "new" knowledge, lack of routine) and facilitators (understanding the biological foundations for SE, knowing it was evidence-based, having reliable criteria for determining the endpoint of SE), the two interventions were developed. 504 dentists participated in the experiment (response rate: $24.9 \%$ ). For both interventions, the 
outcome behavior improved significantly after the intervention $(p<0.001)$, with $29.6 \%$ (guideline) and $17.9 \%$ (tool) changing their behavior towards SE, respectively.

There were no significant differences in the outcome behavior between the two interventions $(p=0.933)$.

Conclusion: Systematically developed behavior-change interventions may be efficacious to improve the uptake of SE.

Clinical significance: Understanding the barriers and facilitators for applying SE facilitates the development of interventions which may be efficacious for changing carious tissue removal.

\section{Zusammenfassung}

Ziele: Für tiefe kariöse Läsionen wird zunehmend ein selektives Exkavationsvorgehen (SE) zum Erhalt der Pulpaintegrität und -vitalität empfohlen. Die Mehrzahl der Zahnärzte wendet SE jedoch nicht als Therapie der Wahl bei tiefen Läsionen an. Das Ziel der Studie war die Identifizierung von Barrieren und fördernden Faktoren zur Anwendung von SE.

Methoden: Es wurden zehn Einzelinterviews mit deutschen Zahnärzten (6 weiblich, 4 männlich, Alter 27-58 Jahre) durchgeführt. Diese Interviews wurden systematisch entlang des Theoretical Domain Frameworks (TDF) entwickelt. Mittels thematischer Analyse unter Einsatz des Behavior Change Wheel (BCW) und der daraus abgeleiteten Methodik (Behavior Change Technique Taxonomy Version 1 (BCTTv1)) wurden zwei verschiedene Interventionen entwickelt. Die Interventionen wurden als Simulationsexperiment zur Verhaltensänderung postalisch an 2452 zufällig ausgewählte deutsche Zahnärzte versandt ( $\mathrm{n}=1226$ / Intervention), wobei die jeweilige Intervention in einem separaten 
verschlossenen Umschlag beigelegt war. Der primäre Ergebnisparameter (simuliertes Verhalten) wurde als Härte des in Pulpanähe belassenen Dentins gemessen. Ausgangsverhalten und Post-Interventionsverhalten wurde jeweils mittels zwölf Fragen erfasst. Für die Vergleiche von Prä- und Post-Interventionsverhalten sowie für die Gruppenvergleiche wurden der Chi-Quadrat-Test und eine Multinomiale-Ordiale Regression durchgeführt.

Ergebnisse: Vier hemmende und vier fördernde Faktoren für SE wurden identifiziert. Die abgeleitete Intervention „Leitlinie“ beinhaltete ein zusammengefasstes Statement der Deutschen Gesellschaft für Zahnerhaltung zur Anwendung von SE. Die abgeleitete Intervention „Werkzeug“ beinhaltete ein simuliertes selbst-limitierendes Winkelstück, welches die Zahnärzte zur Exkavation nutzen können. 504 der kontaktierten Zahnärzte nahmen teil (24.9\% Antwortquote). Die Härte des in Pulpanähe belassenen Dentins war signifikant verschieden im Vergleich von prä- und post-Intervention $(p<0.001)$. In der „Leitliniengruppe“ beließen zusätzliche $29.6 \%$ weiches Dentin, in der „Hilfsmittelgruppe“ waren es $17.9 \%$. Zwischen den Gruppen gab es keine signifikanten Unterschiede $(p=0.933)$.

Fazit: Systematische und auf theoretischen Grundlagen entwickelte Interventionen zur Verhaltensänderung waren wirksam und können in der Implementierung von SE hilfreich sein.

Klinische Relevanz: Das Verstehen von hemmenden und fördernden Faktoren von SE ermöglicht die Entwicklung von Interventionen, die effektiv das Exkavationsverhalten von Zahnärzten verändern können. 


\section{Manteltext}

\section{Einleitung}

Die Kariesexkavation ist im Bereich der Zahnmedizin eine tägliche Routine, der sich in Deutschland Millionen Patienten jährlich unterziehen [1]. Der traditionelle Umgang mit kariösen Läsionen beinhaltete die vollständige mechanische Entfernung von bakteriell infiziertem und demineralisiertem Gewebe, wobei unerheblich war, ob die Läsion in der Peripherie oder nah an der Pulpakammer lag. Die vollständige Entfernung des infizierten Gewebes und der ursächlichen Erreger sollten zu einem längeren Erhalt der Zähne führen sowie die Ausbreitung der Krankheit verhindern.

Dem ursprünglichen Verständnis von Karies als Infektionskrankheit steht die heute geltende ökologische Plaquehypothese [2,3] gegenüber. Sie formuliert, dass die Biofilmaktivität und weniger die Präsenz eines Biofilms an sich entscheidend ist für die Entstehung von Karies. Während in einem physiologischen Biofilm zunächst wenige säurebildende Bakterien vorkommen, verschiebt sich die Spezieszusammensetzung durch Substratzufuhr (Kohlenhydrate) hin zu überwiegend azidogenen und azidurischen (säurebildenden und säuretoleranten) Bakterien, da diese Kohlenhydrate zu Säuren verstoffwechseln und hierdurch andere, nicht säuretoleranten Spezies verdrängen [4]. Säuren lösen Mineralien aus dem Zahn; bei nur kurz anhaltender Demineralisierung kann durch anschließende Mineralisierung durch den Speichel eine Remineralisierung erfolgen. Maturierte Biofilme und häufige Kohlenhydratzufuhr führen jedoch zu einem Netto-Mineralverlust [5], dessen Symptom die kariöse Läsion ist. Heute steht demnach eher die Kontrolle der Biofilmaktivität und weniger die (vollständige) Entfernung der Läsion zur „Heilung“ der Erkrankung im Vordergrund. 
Eine Möglichkeit zur Kontrolle dieser Biofilmaktivität ist eine Unterbindung der Substratzufuhr der Bakterien. In Studien belegte die Gruppe um Mertz-Fairhurst, dass durch eine Unterbindung der Substratzufuhr mittels einer Versiegelung von kariösen Läsionen [6,7] diese Läsionen langfristig arretiert werden können [8]. Gerade im pulpanahen Bereich einer Kavität wird deshalb heute gefordert, kariöses Dentin nicht mehr vollständig auszuräumen, da dies (1) nicht nötig ist, wenn anschließend eine dichte Versiegelung erfolgt und (2) eine solche „vollständige Exkavation“ häufig zu Pulpaexpositionen und -komplikationen führt, mit nachteiligen Folgen für den Zahn [911].

So empfiehlt die Deutsche Gesellschaft für Zahnerhaltung heute eine selektive Exkavation (SE) tiefer, pulpanaher Läsionen zur Pulpaschonung [12]. Im Rahmen von SE erfolgen eine Exkavation bis in das harte Dentin im Kavitätenrandbereich und ein Belassen auch kariös veränderten Dentins im pulpanahen Bereich zur Pulpaschonung vor der definitiven Restauration. Die Beschaffenheit des zu belassenden Dentins ist dabei nicht eindeutig definiert und richtet sich maßgeblich nach der Kavitätentiefe [8].

Obwohl es evidenzbasiert belegt ist, dass SE die Risiken von Pulpaexposition und Pulpakomplikationen reduziert und die Überlebensdauer eines Zahnes erhöht [10], wurde in Umfragestudien mit Zahnärzten festgestellt, dass die befragten Behandler zu etwa $50 \%$ SE ablehnen [13,14]. Unklar bleiben allerdings die diesem Verhalten zugrundeliegenden hemmenden und fördernden Faktoren. Die Identifizierung dieser Faktoren würde es bspw. ermöglichen, Interventionen zur Verhaltensänderung zu entwickeln und damit die Implementierung von SE im Hinblick auf Akzeptanz und Anwendung zu erhöhen. 
Ziel dieser Studie war es daher, hemmende und fördernde Faktoren von SE zu analysieren, Interventionen zur Verhaltensänderung zu entwickeln und diese schließlich auf ihre Wirksamkeit zu testen. Dazu wurden qualitative Interviews und Analysen mit einem quantitativen Verhaltensänderungssimulationsexperiment verknüpft.

\section{Methodik}

Zunächst wurden zehn Einzelinterviews mit Zahnärzten geführt, die anhand der Struktur des Theoretical Domains Framework (TDF) [15] entwickelt wurden. Das TDF ist in 12 Domänen unterteilt. Es basiert auf 128 Konstrukten welche in 33 psychologische Theorien eingebettet sind[16]. Diese strukturierte Zusammenfassung schafft eine vereinfachte Grundlage, mit der ein evidenzbasierter und theoretisch gestützter Zugang zu Implementierungsforschung im Gesundheitsbereich möglich ist. Die 12 Domänen des TDF bilden ab, welche Bereiche für das Entstehen und die Veränderung von Verhalten relevant sind $[16,17]$.

Anhand der zehn ausgewählten Domänen (siehe * in Tabelle 1) des TDF wurden die Fragen für das Interview entwickelt. Um die Beweggründe der Teilnehmer abzufragen und möglichst unbeeinflusste Antworten zu erhalten, wurden offene Fragen gestellt. Der Fragebogen ist dem Appendix zu entnehmen. 
Tab. 1: Domänen des Theoretical Domains Framework (TDF) [15]. Für die Fragebogenentwicklung genutzte Domänen wurden mit einem $\left(^{*}\right)$ markiert.

\begin{tabular}{ll}
\hline Domain & Definition \\
\hline Knowledge* $^{*}$ & An awareness of the existence of something \\
\hline Skills* $^{*}$ & An ability or proficiency acquired through practice \\
\hline Social/ Professional and Identity * & $\begin{array}{l}\text { A coherent set of behaviors and displayed personal qualities of } \\
\text { an individual in a social or work setting }\end{array}$ \\
\hline Beliefs about Capabilities* & $\begin{array}{l}\text { Acceptance of the truth, reality, or validity about an ability, talent, } \\
\text { or facility that a person can put to constructive use }\end{array}$ \\
\hline Optimism* & $\begin{array}{l}\text { The confidence that things will happen for the best, or that desired } \\
\text { goals will be attained }\end{array}$ \\
\hline Beliefs about Consequences* & $\begin{array}{l}\text { Acceptance of the truth, reality, or validity about outcomes of a } \\
\text { behavior in a given situation }\end{array}$ \\
\hline Reinforcement & $\begin{array}{l}\text { Increasing the probability of a response by arranging a dependent } \\
\text { relationship, or contingency, between the response and a given } \\
\text { stimulus }\end{array}$ \\
\hline Intention* & $\begin{array}{l}\text { A conscious decision to perform a behavior or a resolve to act in } \\
\text { a certain way }\end{array}$ \\
\hline Goals* & $\begin{array}{l}\text { Mental representation of outcomes or end states that an } \\
\text { individual wants to achieve }\end{array}$ \\
\hline Memory, Attention and Decision & $\begin{array}{l}\text { The ability to retain information, focus selectively on aspects of } \\
\text { the environment and choose between more alternatives }\end{array}$ \\
\hline Process & $\begin{array}{l}\text { Any circumstance of a person's situation or environment that } \\
\text { discourages or encourages the development of skills and } \\
\text { abilities, independence, social competence, and adaptive } \\
\text { behavior }\end{array}$ \\
\hline Environmental Context and & $\begin{array}{l}\text { Those interpersonal processes that can cause an individual to } \\
\text { change their thoughts, feelings or behaviors }\end{array}$ \\
\hline Resources * & $\begin{array}{l}\text { A complex reaction pattern, involving experiential, behavioral, } \\
\text { and psychological elements, by which the individual attempts to } \\
\text { deal with a personally significant matter or event }\end{array}$ \\
\hline Social Influences & $\begin{array}{l}\text { Anything aimed at managing or changing objectively observed or } \\
\text { measured actions }\end{array}$ \\
\hline Emotion* & \\
\hline &
\end{tabular}


Befragt wurden zehn Zahnärzte. Es wurden zu gleichen Teilen Zahnärzte aus privaten Praxen sowie Universitäten befragt, außerdem wurde eine annähernd gleiche Geschlechteraufteilung angestrebt. Nach Zustimmung zur Datenerhebung und der anonymen Datenverarbeitung wurden die Interviews als Einzelgespräche persönlich oder am Telefon durchgeführt. Die Gespräche wurden aufgezeichnet, transkribiert und ins Englische übersetzt. Anschließen wurden die Antworten kodiert, sortiert und strukturiert gesammelt. Von mehreren Zahnärzten unabhängig voneinander gegebene ähnliche oder gleiche Antworten wurden gruppiert, gezählt und es wurden vier fördernde und vier hemmende Faktoren für die Anwendung der selektiven Exkavation identifiziert.

Ausgehend davon wurden Interventionen zur Erhöhung der Akzeptanz und Anwendung von SE entwickelt. Dazu wurden zunächst unter Zuhilfenahme des Capability Opportunity - Motivation - Behavior Modells (COM-B Model) [18] und des Behavior Change Wheel (BCW) [20] die identifizierten beeinflussenden Faktoren analysiert (Abbildung 1). Das COM-B Modell enthält die jedem Verhalten zu Grunde liegenden Bausteine „Fähigkeit“, „Möglichkeit“ und „Motivation“. „Fähigkeit“ umfasst hierbei sowohl physische als auch psychische Voraussetzungen. „Möglichkeit“ bezeichnet die vom Umfeld gegebenen Rahmenbedingungen, ein Verhalten auszuüben. „Motivation“ beinhaltet innere Überzeugungen, Gefühle und gezielten Entscheidungen, die zu einem Verhalten führen. Diese drei Bausteine beeinflussen nicht nur das Verhalten insgesamt, sondern sich auch gegenseitig und untereinander. Interventionen können daher an einer oder mehreren Stellen angreifen, um Verhalten zu ändern [19]. 
$\square$ Sources of Behavior

$\square$ TDF Domains

$\square$ Intervention Functions

Policy categories

Beh Regulation - Behavioral Regulation

Bel Cap - Beliefs about Capabilities

Bel Cons - Beliefs about Consequences

Cog - Cognitive and Interpersonal Skills

Em - Emotion

Id - Social/Professional Role Identity

Int - Intentions

Memory - Memory, Attention and Decision Process

Opt - Optimism

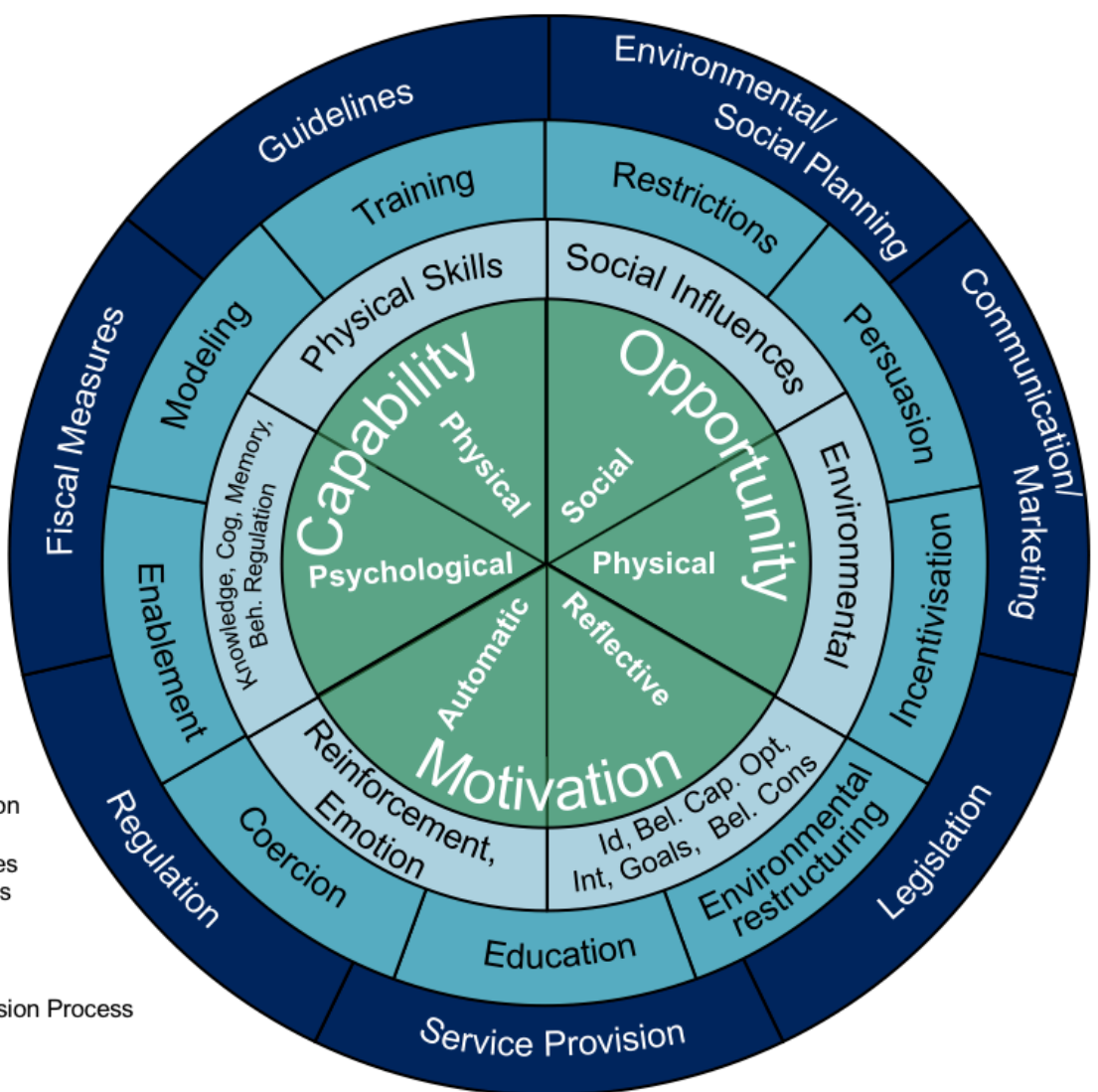

Abb. 1: Das Behavior Change Wheel [20]. Dem COM-B Modell in der Mitte des BCW können in dem zweiten Ring die TDF Domänen zugeordnet werden. Der dritte Ring enthält Interventionsfunktionen, welche das untersuchte Verhalten verändern sollen und mit Techniken zur Verhaltensänderung (Behavior Change Techniques BCT) [19] in der Übersicht der Behavior Change Technique Taxonomy Version 1 (BCTTv1) verknüpfbar sind [21]. In Ring vier sind dann Strategiekategorien abgebildet, die dabei helfen sollen, die Interventionen umzusetzen. 
Zur Entwicklung der Interventionen wurden die identifizierten hemmenden oder fördernden Faktoren schrittweise den Ebenen des BCWs zugeordnet. So wurde bspw. die identifizierte Wissenslücke zu SE (siehe unten) im TDF unter „Knowledge / Skills“ kodiert. Über die Verknüpfung von TDF und COM-B Modell wird dann die Domäne "Capability" des COM-B-Modells zugeordnet. Dem lassen sich drei passenden Interventionswirkungsweisen /-funktionen, nämlich „Enablement“, „Education“ und "Training“ zuordnen. Im vierten Ring wurde schließlich die Strategiekategorie "Guidelines" zugeordnet. Mittels der Behavior Change Technique Taxonomy Version 1 (BCTTv1) [22] wurden daraus zwei Interventionen entwickelt, die jeweils mehrere Faktoren adressieren. Die Entwicklung wird in den Abbildungen 2 und 3 beispielhaft zusammengefasst. 


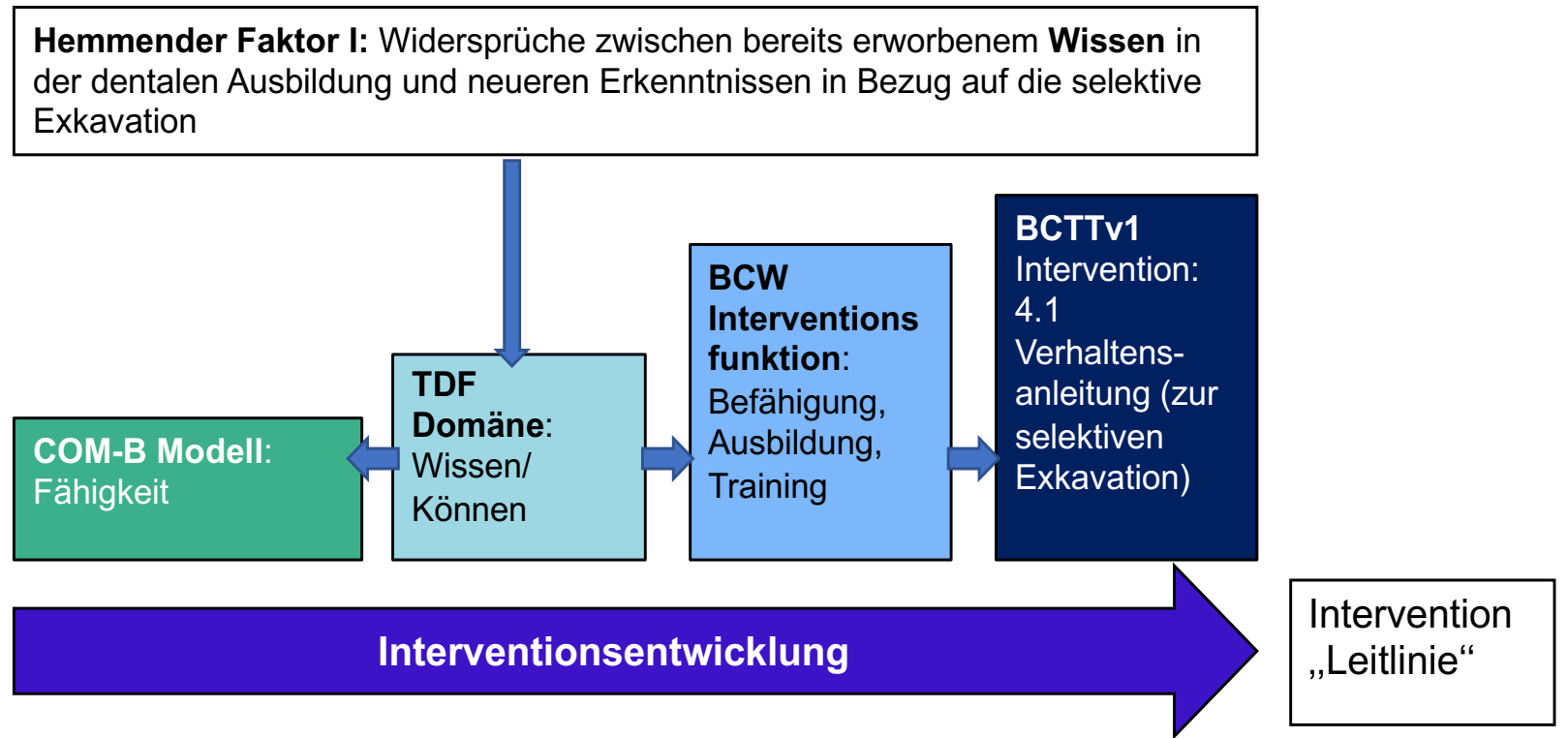

Abb. 2: Darstellung der Interventionsentwicklung für Intervention 1 (Leitlinie). Die identifizierte Wissenslücke zur selektiven Kariesexkavation wurde im TDF unter „Knowledge/ Skills“ kodiert. Dies lässt sich über die Verknüpfung von TDF und COM-B Modell zunächst als „Capability“ in das COM-B-Modell einordnen. Zieht man nun das BCW hinzu, kann man die Kodierung vom ersten Kreis des Rades (COMB-Modell) über den zweiten Ring (TDF-Domäne) in den dritten Ring übertragen. Die drei passenden Interventionswirkweisen waren hierbei „Enablement“, „Education“ und „Training“. Aus dem vierten Ring wurde die Strategie "Guidelines“ zugeordnet. Danach wurde die BCTTv1 genutzt, um die konkreten Interventionsmaßnahmen festzulegen. Unter „Shaping knowledge” wurde aus den möglichen Strategien „4.1 Instruction on how to perform a behavior" ausgewählt. Da es nur bedingt möglich ist, alle tätigen Zahnärzte in praktischen Übungen einzeln fortzubilden, ist eine „Guideline“ (Richtlinie, Stellungnahme, Leitlinie) eine sinnvolle Option. Diese gewählte Strategie wurde dann mit einer zweiten Technik verknüpft: „9.1 Credible Source: present written or visual communication from a credible source in favor of behavior" [21]. Dazu wurde eine gekürzte Stellungnahme der Deutschen Gesellschaft für Zahnerhaltung [12] zur selektiven Kariesexkavation herangezogen. 
Hemmende Faktoren 3+4: Die Schwierigkeit bestehende Routinen aufzugeben und die Angst vor endodontischen Komplikationen; eine fehlende exakte Definition des Endpunktes der selektiven Exkavation

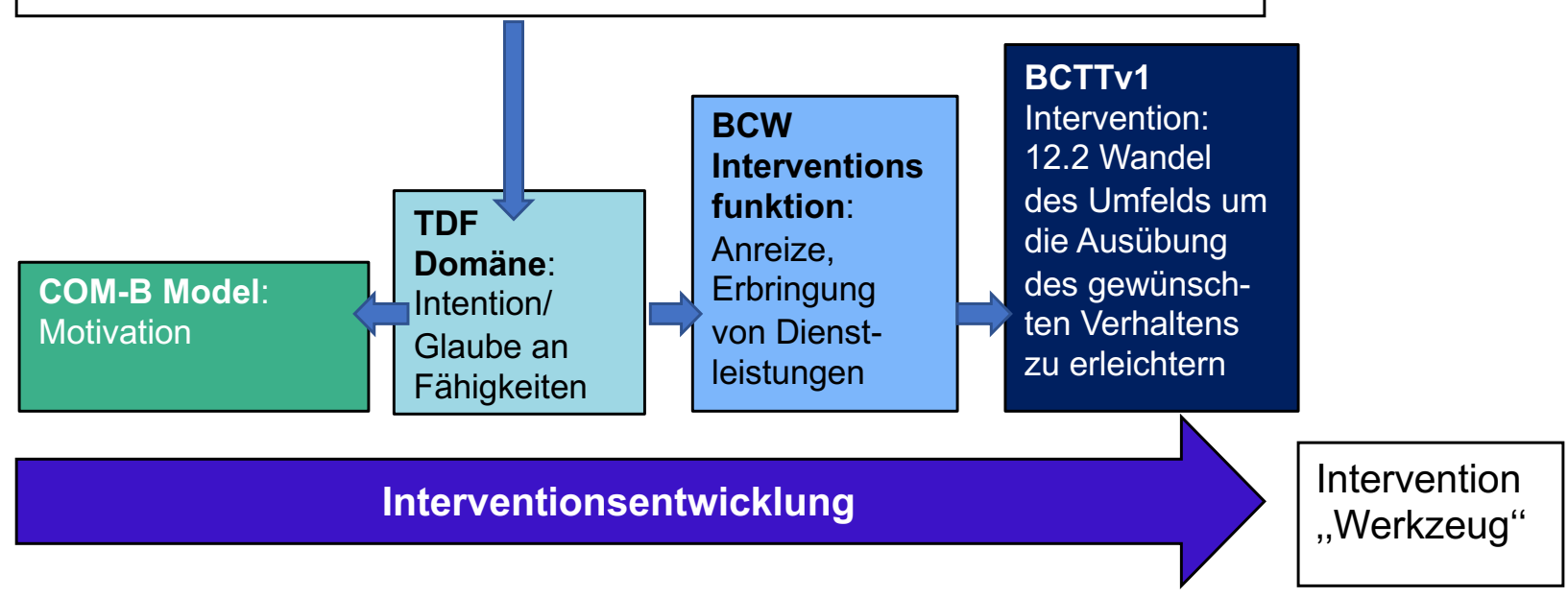

Abb. 3: Darstellung der Interventionsentwicklung, Intervention 2. Die Angst vor Komplikationen sowie die Schwierigkeit bestehende Routinen aufzugeben wurden unter die TDF Domänen „Intention“ und „Beliefs of capabilities“ kodiert. Dies lässt sich über die Verbindung von TDF und COM-B Modell als „Motivation“ in das COM-B Modell einordnen. Nimmt man das BCW hinzu, kann die Codierung in den dritten Ring übertragen werden für die zugehörigen Interventionswirkweisen „Education“, „Coercion“ und „Environmental restructuring“. Aus dem vierten Ring wurde die Strategie „Service provision“ zugeordnet. Mit Hilfe der BCTTV1 wurde die konkrete Interventionsmaßnahme festgelegt. Mittels „3.2 Practical social support" in Verbindung mit „12.1 Restructuring physical environment” und „12.5 Adding objects to the environment" wurde das potentielle Werkzeug entwickelt: Ein Winkelstück, mit dem es Zahnärzten schnell ermöglicht würde, die selektive Exkavation sicher anzuwenden, wobei dabei auch der fehlende Endpunkt als hemmender Faktor eingebunden wäre, da das Winkelstück diesen selbstständig bestimmen würde. Durch die leichte Anwendung würde sich die alte Routine schnell in eine Neue überführen lassen, auch dieser hemmende Faktor wäre damit behoben. Die Intervention basiert auf der hypothetischen Benutzung eines solchen Werkzeuges, da es ein Winkelstück dieser Art bisher nicht gibt. 
Um die entwickelten Interventionen zu testen, wurde ein Simulationsexperiment zur Verhaltensänderung mit in Deutschland praktizierenden Zahnärzten (Gesamt n=2452, aufgeteilt in zwei Gruppen mit jeweils $n=1226 /$ Intervention) durchgeführt. Die Gruppenzuordnung erfolgte zufällig und die Interventionen wurden postalisch zugestellt. Die Zahnärzte wurden hierbei mittels Fragebogen (s. Appendix) zunächst zu Ihrer Einstellung zu SE befragt. Danach wurden sie aufgefordert, den versiegelten zweiten Umschlag mit der Intervention zu öffnen, diese zu lesen und den gleichen Fragebogen zu ihrer Einstellung erneut zu beantworten.

\section{Ergebnisse}

Tab. 2: Teilnehmerdaten ${ }^{*}$ (Anzahl n; beide Gruppen kombiniert sowie stratifiziert nach Gruppen) im Vergleich mit der Zielgruppe (Gesamtheit praktizierender deutscher Zahnärzte) [1].

\begin{tabular}{|c|c|c|c|c|}
\hline & Beide Gruppen & $\begin{array}{l}\text { Gruppe } \\
\text { Leitlinie }\end{array}$ & $\begin{array}{c}\text { Gruppe } \\
\text { Werkzeug }\end{array}$ & Zielgruppe \\
\hline Männlich n (\%) & $265(58.8)$ & $133(56.8)$ & $132(60.8)$ & $39876(55.4)$ \\
\hline Weiblich n (\%) ${ }^{1}$ & $186(41.2)$ & $101(43.2)$ & $85(39.2)$ & $32050(44.6)$ \\
\hline Einzelpraxis n (\%) & $234(52.8)$ & $125(54.1)$ & $109(51.4)$ & $34918(81.9)$ \\
\hline Zweibehandlerpraxis $\mathrm{n}(\%)^{1}$ & $112(25.3)$ & $60(26.0)$ & $52(24.5)$ & $6705(15.7)$ \\
\hline Mehrbehandlerpraxis $\mathrm{n}(\%)^{1}$ & $97(21.9)$ & $46(19.9)$ & $51(24.1)$ & $993(2.4)$ \\
\hline Durchschnittsalter (min.-max.) & $53(25-72)$ & $54(27-77)$ & $52(25-72)$ & $49(22-90)$ \\
\hline
\end{tabular}


Folgende vier fördernde und vier hemmende Faktoren wurden identifiziert:

Fördernde Faktoren:

1. Das Vorhandensein klarer Anwendungsrichtlinien und messbarer, als zuverlässig empfundener Exkavationskriterien.

2. Das Verständnis der biologischen Grundlagen von Karies und der daraus abgeleiteten Exkavationsprinzipien.

3. Die wissenschaftliche Fundierung der SE, insbesondere zum Pulpaschutz.

4. Eine partizipatorische Entscheidungsfindung mit interessierten, informierten Patienten.

Hemmende Faktoren:

1. Der Mangel an vorhandenen bzw. klaren Richtlinien zu SE.

2. Die Widersprüche zwischen erworbenem (Studiums-) Wissen und neuen Erkenntnissen.

3. Mangelnde oder negative Erfahrungen sowie die Sorge vor endodontischen Komplikationen.

4. Die Schwierigkeit, aus vorhandenen Routinen auszubrechen.

Die daraus abgeleiteten Interventionen wurden bereits beschrieben. Diese wurden in einem postalischen Simulationsexperiment überprüft. Bei 2452 kontaktierten Zahnärzten wurde eine Antwortquote von $24.9 \%$ erreicht. Die demographischen Daten der Versuchsgruppe ähnelten denen der Zielgruppe praktizierender deutscher Zahnärzte und waren in den beiden Gruppen nicht signifikant unterschiedlich (Tab. 2). 
In dem Ausgangsfragebogen unterschieden sich die Antworten beider Gruppen nicht signifikant voneinander. Als primärer Ergebnisparameter wurde die simulierte Verhaltensänderung bezogen auf die Härte des belassenen Dentins in Pulpanähe eines vitalen Zahnes genutzt. Dieser primäre Ergebnisparameter änderte sich unabhängig vom Interventionstyp durch die Intervention signifikant $(p<0.001)$; über beide Gruppen hinweg zeigten $23,5 \%$ der Zahnärzte nach der Intervention ein weniger invasives Verhalten bezogen auf die belassene Dentinhärte (29,6\% in der Gruppe „Leitlinie“ und 17,9\% in der Gruppe „Werkzeug“). Zahnärzte in der „Leitliniengruppe“ waren nach der Intervention signifikant positiver gegenüber einer Versiegelung belassener Bakterien eingestellt, während die Gruppe „Werkzeug“ signifikant häufiger den definitiven Endpunkt von SE als unproblematisch empfand.

\section{Diskussion}

Zahnärzte zeigten nach der Anwendung beider Interventionen eine simulierte Verhaltensänderung; die Akzeptanz und simulierte Anwendung von SE stieg signifikant. Die Gruppen unterschieden sich in den Antworten nach der Intervention in so weit, als dass die Zahnärzte aus der „Leitliniengruppe“ durch besseres theoretisches Wissen über die Kariesentstehung das Belassen von Bakterien unter versiegelten Restaurationen positiver betrachteten, das Feststellen des Endpunktes der Exkavation jedoch nach wie vor als schwierig einschätzten. Im Gegenzug stellte für die Interventionsgruppe „Werkzeug“ nach der Intervention die Feststellung des Endpunktes der Exkavation kein Problem mehr dar. Es fehlte die theoretische Komponente, und die Einstellung zum Belassen von Bakterien änderte sich nicht signifikant. 
Der Einsatz gemischt qualitativer und quantitativer Methoden im Rahmen dieser Studie war zielführend. Die vorherige Abfrage der Beweggründe der Zahnärzte ließ eine zielgenaue Entwicklung von Interventionen zu, die wirksam sein könnten, das Verhalten der Studienteilnehmer zu verändern. Durch die konsequente Anwendung der theoretischen Grundlagen TDF, BCW und BCTTV1 konnten die Interventionen strukturiert entwickelt, gut nachvollzogen, ausgewertet und auch miteinander verglichen werden.

Weiterhin ist interessant, dass sich das simulierte Verhalten insgesamt bei beiden Interventionen änderte, die zugrundeliegende Ursachen jedoch offensichtlich verschieden waren. Eine Kombination der beiden getesteten Interventionen könnte demnach noch nachhaltiger und wirksamer sein, da verschiedene verhaltensbestimmende Faktoren adressiert werden. Dabei ist die Intervention „Leitlinie“ verhältnismäßig leicht umzusetzen; sie ist günstig zu entwickeln und administrieren. Die Intervention „Werkzeug“ zielt eher auf den individuellen Zahnarzt ab und kommt mit signifikanten Entwicklungs- und Disseminationsbemühungen daher.

Die vorliegende Arbeit hat durchaus Limitationen. Die Auswahl der interviewten Zahnärzte war nicht repräsentativ und auch die an dem Verhaltensänderungsexperiment teilnehmenden Zahnärzte stellen nur ein Bruchteil der Stichprobengrundgesamtheit dar. Auch wenn anhand von sozio-demographischen Parametern anzunehmen ist, dass die Stichprobe nur bedingt von der Zielpopulation abweicht, ist eine Generalisierbarkeit nur bedingt gegeben [23-25]. Zudem kann nicht sicher davon ausgegangen werden, dass die Teilnehmer die Fragebögen in der richtigen Reihenfolge bearbeitet haben bzw. die Interventionen nicht vorzeitig gelesen wurde. Auch dies könnte zu Verzerrungen in den Ergebnissen führen. Schlussendlich war unser Verhaltensänderungsexperiment nur eine Simulation; klinische Studien wären von Nöten, um wirklich Verhaltensänderungen nachzuweisen. 
Frühere Studien haben jedoch gezeigt, dass Simulationsexperimente bestimmte Ergebnisse aus der Praxis vorhersagen können und bei Entwurf und Auswahl von Interventionen zur Verhaltensänderung nützlich sind [26].

\section{Schlussfolgerungen}

Das Verständnis von fördernden und hemmenden Faktoren zur Anwendung von SE war hilfreich, um theoretisch fundierte Interventionen zur Verhaltensänderung zu entwickeln. Die entwickelten Interventionen waren im Rahmen des durchgeführten Verhaltenssimulationsexperimentes erfolgreich. 


\section{Referenzen}

[1] Kassenzahnärztliche Bundesvereinigung, Jahrbuch 2017, Kassenzahnärztliche Bundesvereinigung, $2017 . \quad$ https://www.kzbv.de/kzbv-jahrbuch2017.media.e21abd6838124f780907f0c25768c989.pdf.

[2] N. Takahashi, B. Nyvad, Caries ecology revisited: Microbial dynamics and the caries process, Caries Res. 42 (2008) 409-418. doi:10.1159/000159604.

[3] N. Takahashi, B. Nyvad, The role of bacteria in the caries process: Ecological perspectives, J. Dent. Res. 90 (2011) 294-303. doi:10.1177/0022034510379602.

[4] Marsh et al., Dental plaque as a biofilm and a microbial community - Implications for treatment, BMC Oral Health. 6 (2006) 185-191. doi:10.1016/j.job.2015.08.002.

[5] O. Fejerskov, E. Kidd, Dental caries: the disease and its clinical management, $\begin{array}{lllll}\text { Second Edi, John Wiley \& Sons, } 2008 . & \end{array}$ https://books.google.de/books?hl=de\&lr=\&id=fZfXWhSmG1UC\&oi=fnd\&pg=PR1\& $\mathrm{dq}=$ Caries+diagnosis:+'A+men+tal+resting+place+on+the+way+to+intervention'\%3F\&ots=OxlaOaXrCN\&sig=Sd vbTc_JdRvA3RyiTNBnvrjhl-A\#v=onepage $\& q=$ time $\&=$ false.

[6] E.J. Mertz-Fairhurst, G.S. Schuster, E.J. Williams, C.W. Fairhurst, Clinical progress of sealed and unsealed caries. Part II: Standardized radiographs and clinical observations, J. Prosthet. Dent. 42 (1979) 633-637.

[7] E.J. Mertz-Fairhurst, J.W. Curtis JR, J.W. Ergle, F.A. Rueggenberg, S.M. Adair, Ultraconservative and Cariostatic Sealed Restaurations: Results at Year 10, J. Am. Dent. Assoc. 129 (2001) 55-66. doi:10.14219/jada.archive.1998.0022.

[8] E.A.M. Kidd, How 'clean' must a cavity be before restoration?, Caries Res. 38 (2004) 305-313. doi:10.1159/000077770.

[9] F. Schwendicke, S. Paris, Kariesexkavation: Ein systematischer Überblick, DZZ. 69 (2014) 456-466. doi:10.3238/dzz.2014.0456.

[10] F. Schwendicke, Unvollständige Kariesentfernung : Eine systematische Übersicht und Meta-Analyse, Z. Evid. Fortbild. Qual. Gesundhwes. 109 (2015) 56-58. doi:10.1016/j.zefq.2015.01.009.

[11] F. Schwendicke, G. Göstemeyer, Understanding dentists' management of deep carious lesions in permanent teeth: a systematic review and meta-analysis, Implement. Sci. 11 (2016) 142-153. doi:10.1186/s13012-016-0505-4.

[12] W. Buchalla, R. Frankenberger, K. Galler, G. Krastl, K.-H. Kunzelmann, S. Paris, 
E. Schäfer, Aktuelle Empfehlungen zur Kariesexkavation, Dtsch. Zahnarzt Zeitschrift. 72 (2017) 484-494.

[13] F. Schwendicke, L. Stangvaltaite, C. Holmgren, M. Maltz, M. Finet, K. Elhennawy, I. Eriksen, T.C. Kuzmiszyn, E. Kerosuo, S. Doméjean, Dentists ' attitudes and behaviour regarding deep carious lesion management: a multi-national survey, Clin. Oral Investig. (2017) 191-198. doi:10.1007/s00784-016-1776-5.

[14] H. Meyer-Lückel, F. Schwendicke, C. Dörfer, S. Paris, Attitudes and Behaviour regarding Deep Dentin Caries Removal : A Survey among German Dentists, Caries Res. 47 (2013) 566-573. doi:10.1159/000351662.

[15] J. Cane, D. O'Connor, S. Michie, Validation of the theoretical domains framework for use in behaviour change and implementation research, Implement. Sci. 7 (2012) 37-53. doi:10.1186/1748-5908-7-37.

[16] S. Michie, M. Johnston, C. Abraham, R. Lawton, D. Parker, A. Walker, Making psychological theory useful for implementing evidence based practice: a consensus approach, Qual. Saf. Heal. Care. 14 (2005) 26-33. doi:10.1136/qshc.2004.011155.

[17] W. Gnich, D. Bonetti, A. Sherriff, S. Sharma, D.I. Conway, Use of the theoretical domains framework to further understanding of what influences application of fluoride varnish to children's teeth: a national survey of general dental practitioners in Scotland, Community Dent. Oral Epidemiol. 43 (2015) 272-281.

[18] H. Westland, I.D. Bos-Touwen, J.C.A. Trappenburg, C.D. Schröder, N.J. de Wit, M.J. Schuurmans, Enhancing physical activity in patients and equipping nurses to deliver a behaviour change intervention, Trials. $18 \quad$ (2017) 79-94. doi:10.1186/s13063-017-1823-9.

[19] S. Michie, M.M. Van Stralen, R. West, The behaviour change wheel: A new method for characterising and designing behaviour change interventions, Implement. Sci. 6 (2011) 42-53.

[20] R. West, S. Michie, Applying the Behavior Change Wheel A very brief guide, Univ. Coll. London. (2015) 1. https://www.ucl.ac.uk/behaviour-change/files/bcwsummary.pdf.

[21] S. Michie, M. Richardson, M. Johnston, C. Abraham, J. Francis, W. Hardeman, M.P. Eccles, J. Cane, C.E. Wood, The Behavior Change Technique Taxonomy (v1) of 93 Hierarchically Clustered Techniques: Building an International Consensus for the Reporting of Behavior Change Interventions, Ann. Behav. Med. 46 (2013) 8195. doi:10.1007/s12160-013-9486-6. 
[22] S. Michie, C.E. Wood, M. Johnston, C. Abraham, J.J. Francis, W. Hardeman, Behaviour change techniques: The development and evaluation of a taxonomic method for reporting and describing behaviour change interventions (a suite of five studies involving consensus methods, randomised controlled trials and analysis of qualitative data), Health Technol. Assess. 19 (2015) 1-187. doi:10.3310/hta19990.

[23] F. Schwendicke, L.A. Foster Page, L.A. Smith, M. Fontana, W.M. Thomson, S.R. Baker, To fill or not to fill: a qualitative cross-country study on dentists' decisions in managing non-cavitated proximal caries lesions, Implement. Sci. 13 (2018) 1-14. doi:10.1186/s13012-018-0744-7.

[24] W. Hardeman, M. Johnston, D. Johnston, D. Bonetti, N. Wareham, A.L. Kinmonth, Application of the Theory of Planned Behaviour in Behaviour Change Interventions: A Systematic Review, Psychol. Heal. 17 (2010) 123-158. doi:10.1080/08870440290013644.

[25] N.P.T. Innes, F. Schwendicke, Restorative Thresholds for Carious Lesions: Systematic Review and Meta-analysis, J. Dent. Res. 96 (2017) 501-508. doi:10.1177/0022034517693605.

[26] D. Bonetti, M. Eccles, M. Johnston, N. Steen, J. Grimshaw, R. Baker, A. Walker, N. Pitts, Guiding the design and selection of interventions to influence the implementation of evidence-based practice: An experimental simulation of a complex intervention trial, Soc. Sci. Med. 60 (2005) 2135-2147. doi:10.1016/j.socscimed.2004.08.072.

[27] L. Atkins, Using the COM-B Model and Behaviour Change Wheel to understand behaviours related to physical activity and design interventions: a short workshop! (slides),

http://www.bhfactive.org.uk/userfiles/Documents/louise_atkins_workshop_211113 Dec2015.pdf. 


\section{Eidesstattliche Versicherung}

„Ich, Linda-Maria Jeggle-Engbert, versichere an Eides statt durch meine eigenhändige Unterschrift, dass ich die vorgelegte Dissertation mit dem Thema: „Changing Dentists' Carious Tissue Removal Behavior: Qualitative Study and Behavioral Change Simulation Experiment." selbstständig und ohne nicht offengelegte Hilfe Dritter verfasst und keine anderen als die angegebenen Quellen und Hilfsmittel genutzt habe. Alle Stellen, die wörtlich oder dem Sinne nach auf Publikationen oder Vorträgen anderer Autoren beruhen, sind als solche in korrekter Zitierung (siehe „Uniform Requirements for Manuscripts (URM)“ des ICMJE (www.icmje.org) kenntlich gemacht. Die Abschnitte zu Methodik (insbesondere praktische Arbeiten, Laborbestimmungen, statistische Aufarbeitung) und Resultaten (insbesondere Abbildungen, Graphiken und Tabellen) entsprechen den URM (s.o.) und werden von mir verantwortet. Mein Anteil an der ausgewählten Publikation entspricht dem, der in der untenstehenden gemeinsamen Erklärung mit dem Betreuer angegeben ist.

Die Bedeutung dieser eidesstattlichen Versicherung und die strafrechtlichen Folgen einer unwahren eidesstattlichen Versicherung ( $\$ 156,161$ des Strafgesetzbuches) sind mir bekannt und bewusst."

Datum: Berlin, den Unterschrift: 


\section{Anteilserklärung an der ausgewählten Publikation}

Jeggle L, Baker S, Schwendicke F

Changing Dentists' Carious Tissue Removal Behavior: Qualitative Study and Behavioral Change Simulation Experiment. J Dent 2018;81(2019):43-51.

\section{Beitrag Linda-Maria Jeggle-Engbert:}

- Literaturrecherche zu der vorliegenden Arbeit;

- Entwicklung, Durchführung, Transkription und Übersetzung der Interviews;

- Datenextraktion, Analyse und Interpretation der Ergebnisse;

- Interventionsentwicklung mit Hilfe der aufgeführten Methodik;

- Durchführung der postalischen Umfrage und Auswertung der Fragebögen;

- Einordnung der Ergebnisse in den aktuellen wissenschaftlichen Stand und Empfehlungen für die weitere Forschung sowie die praktische Anwendung;

- Schreiben des Manuskriptes sowie Erstellen der Abbildungen und Tabellen;

Stempel, Datum und Unterschrift des betreuenden Hochschullehrers 


\section{Auszug aus der Journal Summary List}

(ISI Web of KnowledgeSM)

Journal Data Filtered By: Selected JCR Year: 2017

Selected Editions: SCIE, SSCI Selected Categories: "DENTISTRY, ORAL SURGERY and MEDICINE"

Selected Category Scheme: WoS / Gesamtanzahl: 91 Journale

\begin{tabular}{|c|c|c|c|c|}
\hline Rank & Full Journal Title & $\begin{array}{l}\text { Total } \\
\text { Cites }\end{array}$ & $\begin{array}{l}\text { Journal Impact } \\
\text { Factor }\end{array}$ & $\begin{array}{l}\text { Eigenfactor } \\
\text { Score }\end{array}$ \\
\hline 1 & PERIODONTOLOGY 2000 & 4,308 & 6.220 & 0.004430 \\
\hline 2 & JOURNAL OF DENTAL RESEARCH & 19,305 & 5.380 & 0.020950 \\
\hline 3 & ORAL ONCOLOGY & 8,949 & 4.636 & 0.013760 \\
\hline 4 & CLINICAL ORAL IMPLANTS RESEARCH & 14,065 & 4.305 & 0.016880 \\
\hline 5 & International Journal of Oral Science & 918 & 4.138 & 0.002240 \\
\hline 6 & $\begin{array}{l}\text { JOURNAL OF CLINICAL } \\
\text { PERIODONTOLOGY }\end{array}$ & 13,300 & 4.046 & 0.011660 \\
\hline 7 & DENTAL MATERIALS & 12,466 & 4.039 & 0.012560 \\
\hline 8 & JOURNAL OF DENTISTRY & 8,247 & 3.770 & 0.012020 \\
\hline
\end{tabular}




\section{Druckexemplar der ausgewählten Publikation}

Quellenangabe:

Jeggle L, Baker S, Schwendicke F

Changing Dentists' Carious Tissue Removal Behavior: Qualitative Study and Behavioral Change Simulation Experiment. J Dent 2018;81(2019):43-51.

DOI Verlinkung:

URL http://doi.org/10.1016/j.jdent.2018.12.010 


\section{Appendix}

\section{Appendix Material 1: Interview}

\section{Begrüßung und Erklärung des Ablaufs:}

- Begrüßung: Danksagung und informieren, dass Datenschutz und Anonymität gewahrt bleiben.

- Richtige und falsche Antworten gibt es nicht.

- Fokus des Interviews: Gegenstand von Interesse ist die Entscheidung, tiefe Karies (inneres Dentindrittel) vollständig zu exkavieren oder kariöses, pulpanahes Dentin zur Vitalerhaltung der Pulpa zu belassen (selektive Exkavation) oder zweizeitig zu exkavieren (schrittweise Exkavation).

\section{Demografie:}

- Berufsbezeichnung:

- Berufserfahrung:

- Arbeitsplatz:

- städtisch / öffentlicher Dienst

- Größe des Patientenstammes / Anzahl Helferinnen

- Zeitangabe vor jeder Antwort

\section{Behandlungsablauf:}

a) Wie gehen Sie normalerweise in einem permanenten Zahn vor, der eine pulpanahe Karies bis in das inneren Dentindrittel hat, aber vital und asymptomatisch ist? (Exkavationsstrategie)

b) Wann entscheiden Sie sich nicht weiter zu exkavieren? Wann auf jeden Fall vollständig?

c) Bei einer Eröffnung der Pulpa: Nehmen Sie eine direkte Überkappung vor oder leiten Sie eine Vitalexstirpation (VitE) ein?

d) Wann (Bsp. Größe der Öffnung der Pulpa, Symptome) entscheiden Sie sich sofort zu einer VitE?

e) Wie versorgen Sie pulpanahes gesundes Dentin oder auch das kariöse Restdentin nach der selektiven Exkavation? 


\section{Richtlinien:}

a) Kennen Sie Richtlinien zur Exkavation von kariösen Läsionen oder Lehrmeinungen?

b) Kennen Sie Richtlinien zur vollständigen oder teilweisen Exkavation von kariösen Läsionen?

$\rightarrow$ Falls Richtlinien bekannt weiterfragen:

c) Wie interpretieren Sie die Ihnen bekannten Richtlinien?

d) Wie setzen Sie die Innen bekannten Richtlinien in Ihrer akuten Behandlungssituation um?

e) Helfen Ihnen die Richtlinien bei der Behandlung tiefer Karies?

f) Glauben Sie, die aktuellen Erkenntnisse sind evidenzbasiert?

g) Welche Erfahrungen haben Sie mit Ihrer Auslegung der Richtlinien gemacht?

III. Skills (An ability or proficiency acquired through practice)

a) Nach welchen Kriterien entscheiden Sie, ob sie vollständig exkaviert haben?

b) Nach welchen Kriterien entscheiden Sie, wann Sie ausreichend selektiv exkaviert haben?

c) Welche Fähigkeiten werden benötigt, um eine schrittweise Kariesexkavation vorzunehmen?

IV. Beliefs about Capabilities (acceptance of the truth, reality or validity about an ability, talent or facility, that a person can put to constructive use)

a) Wie schwierig ist es für Sie, eine Kavität vollständig zu exkavieren und entsprechend zu versorgen? (Konkreter: Skala I bis VI)

b) Wie schwierig ist es für Sie, eine Kavität nicht komplett zu exkavieren, sondern im pulpanahen Dentin zu stoppen, kariöses Dentin zurück zu lassen und anschließend zu versorgen? (Skala 1 bis VI)

c) Welche Probleme sind dabei bisher aufgetreten?

$\rightarrow$ Bei selektiver Exkavation?

$\rightarrow$ Bei vollständiger Exkavation? (Pulpitis, ...)

Falls Probleme:

$\rightarrow$ Was genau macht das selektive Exkavieren Ihrer Meinung nach schwierig? 
Zeit:

$\rightarrow$ Was erleichtert evtl. die Versorgung des Zahnes nach selektiver Exkavation?

$\rightarrow$ Was genau macht das vollständige Exkavieren schwierig?

$\rightarrow$ Was erleichtert die Versorgung des Zahnes nach vollständiger Exkavation?

V. Optimism (the confidence that things will happen for the best or that desired goals will be attained)

a) Wie optimistisch sind Sie, die Versorgung eines Zahnes nach vollständiger / selektiver / schrittweiser Kariesexkavation gut zu beherrschen?

Zeit:

VI. Beliefs about Consequences (acceptance of the truth, reality or validity about outcomes of a behavior in a given situation)

a) Was sind in Ihren Augen die Vorteile der vollständigen / selektiven / schrittweise Exkavation?

b) Was sind Nachteile?

c) Was sind Anreize?

d) Glauben Sie, die Vorteile wiegen jeweils die Nachteile auf?

e) Denken Sie, die selektive Exkavation verhindert eine WKB?

f) Wird dadurch der Zahn evtl. länger erhalten?

g) Glauben Sie, die selektive Entfernung von pulpanahem kariösen Restdentin führt zu einem Voranschreiten der Karies?

h) Glauben Sie, die selektive Entfernung von pulpanahem kariösen Restdentin führt zu weniger dichten bzw. belastbaren und damit weniger langlebigen Restaurationen?

i) Glauben Sie, die selektive Entfernung von pulpanahem kariösen Restdentin führt zu einer anhaltenden Schädigung und Intoxikation der Pulpa durch Bakterien?

j) Glauben Sie, die selektive Entfernung von pulpanahem kariösen Restdentin führt zur besseren Mundgesundheit des Patienten?

k) Was sind Ihrer Meinung nach, die Konsequenzen für den Patienten bzw. den Zahn, wenn schrittweise exkaviert wird?

I) Denken Sie, die vollständige Exkavation verhindert eine WKB?

m) Wird dadurch der Zahn evtl. länger erhalten? 
n) Glauben Sie, die vollständige Entfernung von pulpanahem kariösen Restdentin führt zu weniger dichten bzw. belastbaren und damit weniger langlebigen Restaurationen?

o) Glauben Sie, die vollständige Entfernung von pulpanahem kariösen Restdentin führt zur besseren Mundgesundheit des Patienten?

p) Was sind die Konsequenzen für den Patienten bzw. den Zahn, wenn vollständig exkaviert wird?

VII. Emotions (a complex reaction pattern, involving experiential, behavioral and physiological elements, by which the individual attempts to deal with a personally significant matter or event)

a) Machen Sie sich Sorgen um die Patienten bei denen Sie vollst. / selekt. / schrittw. exkaviert haben? Und beeinflusst das Ihre Behandlung?

b) Ist die Therapie der vollst. / selekt. / schrittw. Exkavation etwas, hinter dem Sie wirklich stehen?

VIII. Memory, Attention and Decision Process (the ability to retain information, focus selectively on aspects of the environment and choose between two or more alternatives)

a) Fällt es Ihnen leicht, sich den Ablauf der besprochenen Behandlung zu merken Durchführung?

IX. Environmental Context and Resources (any circumstance of a person's situation or environment that discourages or encourages the development of skills and abilities, independence, social competence and adaptive behavior)

a) Welche wirtschaftlichen Faktoren beeinflussen Ihre Entscheidung hinsichtlich der vollst. / selekt. / schrittw. Kariesexkavation?

b) In welchem Ausmaß beeinflussen praxisimmanente Faktoren Ihre Motivation eine selektive / schrittw. Exkavation vorzunehmen?

a. Physikalische Ressourcen: Schulung des Praxispersonals

b. Finanzen: Zeitaufwand, Zuzahlungsbereitschaft der Patienten

c. Erwartungen, Haltung der Kollegen, Einstellungen zur Methode (prompt: which factors act as barriers and which as facilitators?) 
c) Welche patientenimmanenten Faktoren beeinflussen Ihre Motivation / Entscheidung?

d) Kooperation, Erwartungen, Compliance, Mundhygiene, etc.

e) Welche nicht äußeren Faktoren beeinflussen Ihre Entscheidung für oder gegen die besprochene Methode?

f) Welche äußeren Faktoren beeinflussen Ihre Entscheidung? (z.B. Kassenregularien, Gutachterentscheidungen, keine Gebührenposition für schrittweise Exkavation, Garantiezeit für Restauration (nicht für Pulpa), unklare Rechtslage, ...)

g) Glauben Sie es sei möglich vor der Restauration, alle bis ins pulpanahe Dentindrittel vorgedrungenen Kariesstellen selektiv zu exkavieren? (Ist das immer möglich, wann nicht?)

X. Behavioral Regulation (anything aimed at managing or changing objectively observed or measured actions)

a) Denken Sie, Sie sollten nach (lege artis / Lehrmeinung / aktuellem Forschungsstand) die Methode nutzen?

XI. Goals and Intentions (a conscious decision to perform a behavior or resolve to act in a certain way)

a) Ist die Vitalerhaltung der Pulpa durch die selektive / schrittw. Exkavation eine Methode der Versorgung, die Sie in der Zukunft, wann immer anwendbar, einsetzen möchten?

XII. Social Influences (those interpersonal processes that can cause individuals to change their thoughts, feelings or behaviors)

a) In wie weit werden Sie beeinflusst von Patienten oder Ihrem Umfeld, die Methode der selektiven / schrittw. Exkavation zu nutzen?

b) Ist die selektive Exkavation eine Methode die sich Ihre Patienten wünschen? Spielt die Vitalerhaltung durch diese Methode eine Rolle im Auge der Patienten und wünschen Sie sich diese auf Grund dessen? Was spielt noch eine Rolle für Ihre Patienten?

c) Unterstützen andere Kollegen Sie in der besprochenen Form der Versorgung? 
XIII. Social/ professional Role and Identity (A coherent set of behaviors and displayed personal qualities of an individual in a social or work setting)

a) Denken Sie die Vitalerhaltung der Pulpa durch eine selektive /schrittw. Exkavation ist ein entscheidender Teil Ihrer zahnärztlichen Tätigkeit? 


\section{Appendix Material 2: Direkte Zitate der geführten Interviews (übersetzt)}

\begin{tabular}{|c|c|c|}
\hline Domain & $\begin{array}{l}\text { Barrier } \\
\text { facilitator }\end{array}$ & Dentists' quotes \\
\hline \multirow[t]{3}{*}{$\begin{array}{l}\text { Knowledge/ } \\
\text { Resources }\end{array}$} & $\begin{array}{l}\text { Lack of explicit } \\
\text { guidelines }\end{array}$ & $\begin{array}{l}\text { - Guidelines no. But I know what's said in further trainings here. } \\
\text { But no, not a guideline. (Dentist No. 1) } \\
\text { - Especially guidelines, if there was a guideline on the } \\
\text { homepage of the DGZMK, I have to admit, I don't know it at } \\
\text { the moment (Dentist No. 4) } \\
\text { - But there are also some published things from whatever } \\
\text { institutions that are not accessible easily, why would the } \\
\text { dentist read that. (Dentist No. 3) } \\
\text { - (Do you know guidelines of SE?) No. (Dentist No. 6) } \\
\text { - (Do you know guidelines of SE? In the sense of principles?) } \\
\text { Oh no. (Dentist No. 7) } \\
\text { - (Do you know guidelines for excavation?) Well surely not the } \\
\text { new ones. No, l'd say... I did not improve my skills... } \\
\text { No. (Dentist No. 9) }\end{array}$ \\
\hline & $\begin{array}{l}\text { Discrepancies } \\
\text { between } \\
\text { knowledge and } \\
\text { latest research } \\
\text { on SE }\end{array}$ & $\begin{array}{l}\text { - If I'm able to excavate fully I think it prevents root canal } \\
\text { treatment (RCT) rather than selective excavation. (Dentist No. } \\
\text { 4) } \\
\text { - That means, recording to the latest findings, it is allowed, to } \\
\text { leave slightly a bit of caries. There I don't proceed so explicitly. } \\
\text { (Dentist No. 10) } \\
\text { - So, we learned, the color is not essential, only the hardness of } \\
\text { the tissues, that is my guideline. In my education I grew up } \\
\text { with the preparation rules of Black, to open extensively in order } \\
\text { to see the whole caries. (Dentist No. 5) } \\
\text { - I would say I still know the guidelines from Münster, which I } \\
\text { learned in my studies. I don't know any further guidelines } \\
\text { (Dentist No. 10) }\end{array}$ \\
\hline & $\begin{array}{l}\text { Uncertainty of } \\
\text { carious tissue } \\
\text { removal } \\
\text { endpoint }\end{array}$ & $\begin{array}{l}\text { - I ... (stop excavation) ... if there is no more soft tissue. } \\
\text { Until I notice it is not a rough structure anymore, but everything } \\
\text { is smooth. (Dentist No. 4) } \\
\text { - I the end I judge it according to the brilliance. (Dentist No. 6) } \\
\text { - I excavate until I have the feeling now it's getting hard. And } \\
\text { then, when I think it is hard, then I test it with a dental probe. } \\
\text { (Dentist No. 9) } \\
\ldots \text { normally hard on probing, sometimes detectors for caries if } \\
\text { one is not really sure, but rather infrequently. And, of course } \\
\text { visually, often one can see it already. (Dentist No. 7) } \\
\text { The outer areas, in the enamel and the adjoining dentin, also } \\
\text { cervical should be hard and clanking, coming closer to the } \\
\text { pulp I would be more generous and leave scratchable dentin } \\
\text { behind. (Dentist No. 5) }\end{array}$ \\
\hline $\begin{array}{l}\text { Beliefs } \\
\text { about } \\
\text { Capabilities/ } \\
\text { Emotions }\end{array}$ & $\begin{array}{l}\text { Fear of } \\
\text { endodontic } \\
\text { treatment/ } \\
\text { Avoiding of } \\
\text { endodontic } \\
\text { treatment }\end{array}$ & $\begin{array}{l}\text { - (With) SE, that one can retard the endodontic treatment, even } \\
\text { prevent it completely. (Dentist No. 6) } \\
\text { - If I'm in that zone with SE, where I prevent RCT this would be } \\
\text { again an advantage. (Dentist No. 9) } \\
\text { - So, the advantages of SE would of course be, that we are able } \\
\text { to keep the tooth vital, which we had otherwise to treat with } \\
\text { RCT. In the end it counts to preserve endodontic treatment. } \\
\text { (Dentist No. 10) } \\
\text { - I am happy if I can avoid endodontics. (Dentist No. 3) } \\
\text { - On the other hand, the WKB is also a risk. (Dentist No. 2) }\end{array}$ \\
\hline $\begin{array}{l}\text { Skills and } \\
\text { Capability }\end{array}$ & $\begin{array}{l}\text { Rosehead bur } \\
\text { handling }\end{array}$ & $\begin{array}{l}\text { - Somewhat hard, but is still l'd say centrally a bit discolored, } \\
\text { then I take a smaller rosehead bur and take of the } \\
\text { discoloration. (Dentist No. 9) }\end{array}$ \\
\hline
\end{tabular}




\begin{tabular}{|c|c|c|}
\hline & & $\begin{array}{l}\text { - I take the rosehead bur and excavate with } 5600 \text { rotations/min } \\
\text { with the green bend. } \\
\text { I check visually if it shines and finally with the rosehead bur if } \\
\text { it chips. (Dentist No. } 6 \text { ) }\end{array}$ \\
\hline $\begin{array}{l}\text { Beliefs } \\
\text { about } \\
\text { Capabilities }\end{array}$ & $\begin{array}{l}\text { SE prevents } \\
\text { endodontic } \\
\text { treatment }\end{array}$ & $\begin{array}{l}\text { - Yes, (total excavation) the risk of opening the nerve and the } \\
\text { unnecessary endodontic treatment. The advantages I see (in } \\
\text { SE) is frankly the attempt of avoiding a pulp opening and } \\
\text { endodontic treatment (Dentist No.10) } \\
\text { - I Well selectively in any case that the RCT is avoided. (Dentist } \\
\text { No. 2) } \\
\text { - (Does SE prohibit endodontics?) I think so. (Dentist No. 3) } \\
\text { - With SE, that one can postpone the endodontic treatment, } \\
\text { even prevent it completely. (Dentist No. 5) } \\
\text { - (Do you think SE prevents RCT?) Yes.... The advantages of } \\
\text { SE are clearly given for the long-term tooth preservation. } \\
\text { (Dentist No. 8) }\end{array}$ \\
\hline $\begin{array}{l}\text { Intention (A } \\
\text { conscious } \\
\text { decision to } \\
\text { stick to well- } \\
\text { known } \\
\text { procedure) }\end{array}$ & $\begin{array}{l}\text { Lack of } \\
\text { experience with } \\
\text { SE/ lack of } \\
\text { routines due to } \\
\text { other routines } \\
\text { they have } \\
\text { already }\end{array}$ & $\begin{array}{l}\text { - And if everything that is not free of caries, where I chose the } \\
\text { SE approach, the resilience drops. I don't know how much, I } \\
\text { cannot estimate it properly. I don't have enough experience } \\
\text { yet. (Dentist No. 8) } \\
\text { - I think I right now I could not decide in favor of one form of } \\
\text { excavation, because I have to little experience for it. (Dentist } \\
\text { No. 6) } \\
\text { - I would always initiate a direct capping, especially the younger } \\
\text { the patient is. And if I am relatively sure that I did not bring to } \\
\text { much material contaminated with pathogenic germs into the } \\
\text { opened pulp. (Dentist No. 3) } \\
\text { Normally I try to excavate fully, but in exceptional cases I used } \\
\text { my gut instincts depending on how much caries is left. (Dentist } \\
\text { No. 4) }\end{array}$ \\
\hline $\begin{array}{l}\text { Resources/ } \\
\text { Knowledge }\end{array}$ & $\begin{array}{l}\text { Precise } \\
\text { guidelines and } \\
\text { reliable } \\
\text { guidelines } \\
\text { facilitate SE }\end{array}$ & $\begin{array}{l}\text { - (Do the guidelines help when treating a deep carious lesion?) } \\
\text { Yes, it is helpful and provides certainty for me, because after } \\
\text { all I learned it differently before (Dentist No. 5) } \\
\text { - If the guideline seems plausible to me I try to implement it as } \\
\text { good as possible, because it is based on scientific basics. } \\
\text { (Dentist No. 8) } \\
\text { - Yes, it is (guideline) helpful and provides certainty (Dentist No. } \\
\text { 2) } \\
\text { I have to work along the guidelines, I have evidence about all } \\
\text { my further trainings. According to this I can justify my work. } \\
\text { Yes, also the selective excavation. (Dentist No. 10) }\end{array}$ \\
\hline $\begin{array}{l}\text { Social } \\
\text { influences }\end{array}$ & $\begin{array}{l}\text { Participatory } \\
\text { decision making } \\
\text { with the well- } \\
\text { informed patient }\end{array}$ & $\begin{array}{l}\text { - I believe things have to be well explained to patient, because } \\
\text { the patient will in the first instance ask 'How can the dentist } \\
\text { leave caries behind', because otherwise the patient will not be } \\
\text { able to understand. He might misunderstand and think that } \\
\text { one is the bad, incompetent dentist. (Dentist No. 10) } \\
\text { - One has to explain things to the patient of course, and explain } \\
\text { also that it is not always successful this SE. But FE is neither. } \\
\text { (Dentist No. 8) } \\
\text { - I always explain things to the patients. (Dentist No. 2) } \\
\text { - Except I have a dentist (as patient) who is well informed } \\
\text { theoretically, then I would explain it to him, and show him } \\
\text { evidence-based dentistry, then he should probably be } \\
\text { convinced. (Dentist No. 3 }\end{array}$ \\
\hline
\end{tabular}




\section{Appendix Material 3: Ergebnisse der Multinomial-Ordinalen Regression.}

Das Modell prognostizierte das Ergebnis ( $n=381$ Teilnehmer; vollständige Fallanalyse), die simulierte Verhaltensänderung nach der Intervention (gemessen in Dentinhärte in Pulpanähe), signifikant besser als das Intercept-Only Model $(p=0.004)$. Keine der Prädiktorvariablen prognostizierte jedoch signifikant das exakte Ergebnisniveau. $(p>0.05)$. Als Vergleichslevel der Ergebnisse wurde ,hartes Dentin“ genutzt $(n=29)$.

\begin{tabular}{|c|c|c|c|}
\hline Outcome level & Parameter & $\mathrm{OR}, 95 \% \mathrm{Cl}$ & $p$-value \\
\hline \multirow[t]{4}{*}{ Firm dentin ( $n=217)$} & Group Guideline (ref.: Tool) & $0.87,0.49-2.32$ & 0.882 \\
\hline & Age (years) & $1.01,0.91-1.12$ & 0.835 \\
\hline & Year of exam (years) & $1.01,0.91-1.12$ & 0.872 \\
\hline & Male sex (ref: female) & $0.92,0.40-2.08$ & 0.833 \\
\hline \multirow[t]{4}{*}{ Soft dentin ( $n=83$ ) } & Group Guideline (ref.: Tool) & $0.93,0.39-2.19$ & 0.859 \\
\hline & Age (years) & $0.94,0.84-1.06$ & 0.340 \\
\hline & Year of exam (years) & $0.99,0.88-1.12$ & 0.936 \\
\hline & Male sex (ref: female) & $0.82,0.33-2.01$ & 0.663 \\
\hline \multirow{4}{*}{$\begin{array}{l}\text { Hardness does not matter } \\
(n=52)\end{array}$} & Group Guideline (ref.: Tool) & $1.15,0.46-2.89$ & 0.766 \\
\hline & Age (years) & $0.89,0.78-1.02$ & 0.104 \\
\hline & Year of exam (years) & $0.93,0.81-1.06$ & 0.282 \\
\hline & Male sex (ref: female) & $1.37,0.52-3.65$ & 0.524 \\
\hline
\end{tabular}




\section{Appendixmaterial 4:}

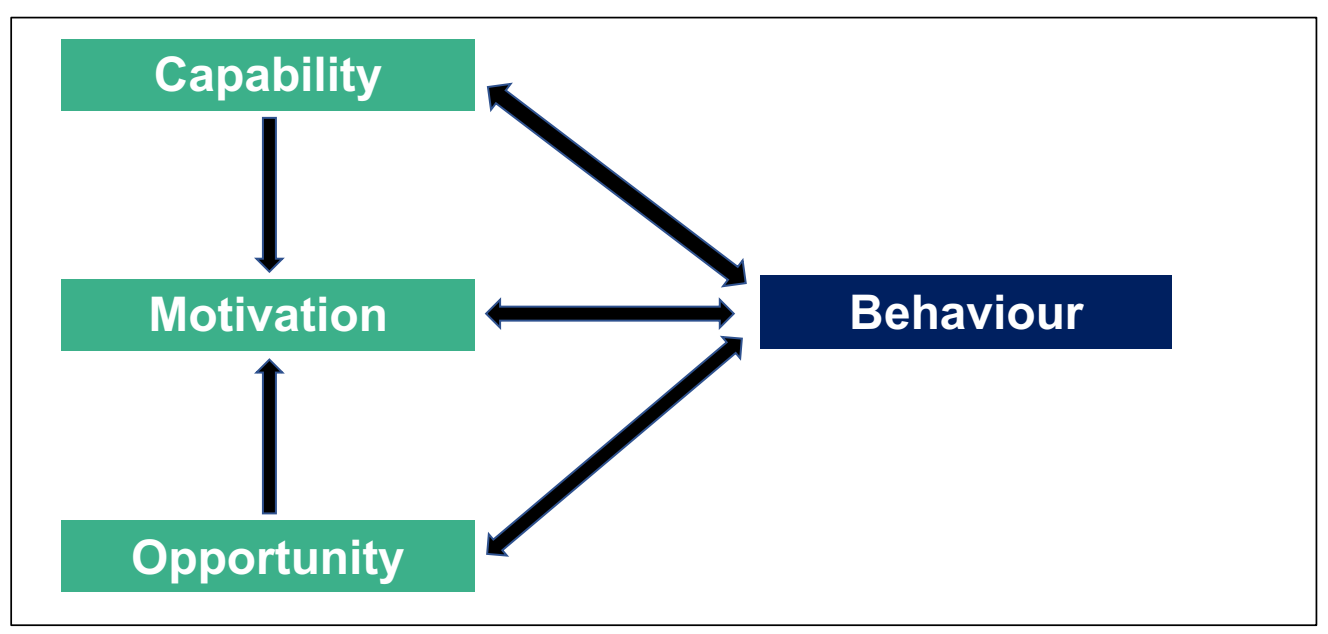

Abb. 4: Das COM-B Modell. Capability: Die Fähigkeit psychische oder physisch ein Verhalten auszuüben. Opportunity: Die Möglichkeit die das physische und soziale Umfeld bieten, um ein Verhalten auszuüben. Motivation: Reflektierende und unbewusste Mechanismen, die Verhalten fördern oder verhindern [18]. Um Verhalten zu ändern, müssen eine oder mehrere Komponenten durch eine Intervention geändert werden. Das COM-B Modell bildet die Mitte des BCW (siehe Abb. 3). 


\title{
Appendix Material 5: Fragebögen mit den Intervention A und B
}

Charité | Campus Benjamin Franklin | 12200 Berlin

\author{
CharitéCentrum 3 für Zahn-, Mund-, und Kieferheilkunde \\ Charité - Universitä tsmedizin Berlin \\ Abteilung für Zahnerhaltung und Präventivzahnmedizin \\ Direktor: Prof. Dr. Sebastian Paris \\ Standort: Assmannshauser-Str. 4-6 | 14197 Berlin \\ Korrespondenz: ZÄ Linda-Maria Jeggle \\ Tel. 030/450562 358 \\ Fax 030/450 7562332 \\ Linda-Maria.Jeggle@charite.de \\ https://zahnerhaltung.charite.de/ \\ Berlin, 05. März 2018
}

\section{Umfrage für Zahnärztinnen und Zahnärzte zur Exkavation tiefer kariöser Läsionen}

Liebe Kollegin, lieber Kollege,

in der vorliegenden Umfrage untersuchen wir, wie deutsche Zahnärztinnen und Zahnärzte tiefe kariöse Läsionen in vitalen Zähnen behandeln. Wir würden uns freuen, wenn Sie sich ein paar Minuten Zeit nehmen und den vorliegenden Fragebogen ausfüllen könnten.

Der Fragebogen enthält 12 Fragen. Die Beantwortung wird etwa 10 Minuten dauern. Wir bitten Sie, alle Fragen sorgfältig zu lesen und zu beantworten. Es gibt weder richtige noch falsche Antworten. Der erste Teil des Bogens erfragt Ihre jetzige Einstellung und Ihr Verhalten bei der Therapie der geschilderten Kariesläsionen. Im zweiten Teil untersuchen wir, ob Sie dieses Verhalten unter bestimmten Umständen ändern würden, dafür liegt ein gesonderter Umschlag bei. Bitte öffnen Sie diesen erst nach Bearbeitung des ersten Teils. Er enthält einen kurzen Text und den zweiten Teil der Fragen. Nach Beantwortung aller 12 Fragen können Sie alle Seiten in den beigelegten Rückumschlag geben und kostenfrei an uns zurücksenden. Die erhobenen Daten werden anonymisiert evaluiert und nach datenschutzrechtlichen Vorschriften verarbeitet.

Wir bedanken uns für Ihre Mühe im Voraus. 
1. Welches Exkavationskriterium nutzen Sie und wann stoppen Sie die Exkavation im pulpanahen Dentindrittel bei einem vitalen Zahn, der keine Anzeichen einer irreversiblen Pulpitis aufweist? (Bitte in jeder Spalte einmal ankreuzen)

\begin{tabular}{|c|c|c|c|c|c|}
\hline \multicolumn{2}{|c|}{ Härte } & \multicolumn{2}{|c|}{ Farbe } & \multicolumn{2}{|c|}{ Feuchtigkeit } \\
\hline $\begin{array}{l}\text { Weiches } \\
\text { Dentin }\end{array}$ & $\square$ & $\begin{array}{l}\text { Stark } \\
\text { verfärbtes } \\
\text { Dentin }\end{array}$ & $\square$ & $\begin{array}{l}\text { Der } \\
\text { Kavitätenboden } \\
\text { ist stark feucht }\end{array}$ & $\square$ \\
\hline $\begin{array}{l}\text { Festes, noch } \\
\text { spanendes } \\
\text { Dentin }\end{array}$ & $\square$ & $\begin{array}{l}\text { Leicht } \\
\text { verfärbtes } \\
\text { Dentin }\end{array}$ & $\square$ & $\begin{array}{l}\text { Der } \\
\text { Kavitätenboden } \\
\text { ist leicht feucht }\end{array}$ & $\square$ \\
\hline $\begin{array}{l}\text { Hartes } \\
\text { Dentin } \\
\text { (Sondentest) }\end{array}$ & $\square$ & $\begin{array}{l}\text { Unverfärbtes } \\
\text { Dentin }\end{array}$ & $\square$ & $\begin{array}{l}\text { Der } \\
\text { Kavitätenboden } \\
\text { ist trocken }\end{array}$ & $\square$ \\
\hline $\begin{array}{l}\text { Die Härte ist } \\
\text { nicht wichtig }\end{array}$ & $\square$ & $\begin{array}{l}\text { Die Farbe ist } \\
\text { nicht wichtig }\end{array}$ & $\square$ & $\begin{array}{l}\text { Die } \\
\text { Feuchtigkeit ist } \\
\text { nicht wichtig }\end{array}$ & $\square$ \\
\hline
\end{tabular}

Wie sehr stimmen sie den folgenden Aussagen zu:

2. Bei einem vitalen Zahn, der keine Anzeichen einer irreversiblen Pulpitis aufweist, exkaviere ich pulpanah zurückhaltender als in der Kavitätenperipherie.

\begin{tabular}{|l|c|c|c|}
\hline Stimme zu & Stimme eher zu & $\begin{array}{c}\text { Stimme } \\
\text { teilweise zu }\end{array}$ & $\begin{array}{c}\text { Stimme eher } \\
\text { nicht zu }\end{array}$ \\
\hline$\square$ & $\square$ & $\square$ & \\
\hline
\end{tabular}

3. Kariesbakterien sollten vollständig - auch in Pulpanähe - entfernt werden, da die Karies sonst voranschreitet.

\begin{tabular}{|c|c|c|c|}
\hline Stimme zu & Stimme eher zu & $\begin{array}{c}\text { Stimme } \\
\text { teilweise zu }\end{array}$ & $\begin{array}{c}\text { Stimme eher } \\
\text { nicht zu }\end{array}$ \\
\hline$\square$ & $\square$ & $\square$ & $\square$ \\
\hline
\end{tabular}




\begin{tabular}{|c|c|c|c|c|}
\hline Stimme zu & Stimme eher zu & $\begin{array}{l}\text { Stimme } \\
\text { teilweise zu }\end{array}$ & $\begin{array}{c}\text { Stimme eher } \\
\text { nicht zu }\end{array}$ & Stimme nicht zu \\
\hline$\square$ & $\square$ & $\square$ & $\square$ & $\square$ \\
\hline
\end{tabular}

5. Wissenschaftliche Stellungnahmen und Empfehlungen haben einen Einfluss auf meine Behandlung.

\begin{tabular}{|c|c|c|c|c|}
\hline Stimme zu & Stimme eher zu & $\begin{array}{c}\text { Stimme } \\
\text { teilweise zu }\end{array}$ & $\begin{array}{c}\text { Stimme eher } \\
\text { nicht zu }\end{array}$ & Stimme nicht zu \\
\hline$\square$ & $\square$ & $\square$ & $\square$ \\
\hline
\end{tabular}

6. Ich exkaviere immer, auch pulpanah, bis ins harte Dentin, weil ich andere Dentinqualitäten nicht zuverlässig überprüfen kann.

\begin{tabular}{|c|c|c|c|}
\hline Stimme zu & Stimme eher zu & $\begin{array}{c}\text { Stimme } \\
\text { teilweise zu }\end{array}$ & $\begin{array}{c}\text { Stimme eher } \\
\text { nicht zu }\end{array}$ \\
\hline$\square$ & $\square$ & $\square$ & Stimme nicht zu \\
\hline
\end{tabular}

Bitte öffnen Sie nun den Umschlag mit dem zweiten Teil der Befragung, und beantworten Sie die enthaltenen Fragen nach dem Lesen eines kurzen Textes. 


\section{Im Folgenden lesen Sie eine Zusammenfassung der wissenschaftlichen Mitteilung der Deutschen Gesellschaft für Zahnerhaltung (DGZ) zum aktuellen Stand der Kariesexkavation im Rahmen der Füllungstherapie 2017. ${ }^{1}$}

Die Empfehlungen beziehen sich auf vitale Zähne der permanenten Dentition, die weder klinisch noch röntgenologisch Symptome einer irreversiblen Pulpitis oder apikalen Parodontitis aufweisen. Sie berücksichtigen den aktuellen Kenntnisstand der Ätiologie, Pathogenese und Therapie von kariösen Läsionen. Folgende Aspekte können zusammengefasst werden:

1. Ursache für die Entstehung von Karies und kariösen Läsionen ist die Etablierung eines Ungleichgewichtes im Zahnbiofilm zugunsten kariogener Bakterien. Diese verstoffwechseln Kohlenhydrate zu Säuren, und können durch die anschließende pH-Wertsenkung physiologische Bakterien verdrängen. Ohne Kohlenhydrate käme es nicht zu Karies und kariösen Läsionen².

2. Mehrere Studien zeigten, dass unter einer dichten Restauration verbliebene Läsionen nicht voranschreiten, da die versiegelten Bakterien kaum noch Zugang zu weiteren Kohlenhydraten haben. Eine Kariesprogression kann nicht mehr stattfinden. So versiegelten Mertz-Fairhurst et al. Karies für einen Zeitraum von 10 Jahren und konnten keine nennenswerten Läsionsprogressionen zeigen ${ }^{3}, 4$. Auch andere Arbeitsgruppen bestätigten diese Ergebnisse ${ }^{5}$. Demnach kann kariöses Dentin unter einer Restauration belassen und versiegelt werden, ohne dass die Läsion voranschreitet.

3. Auf Karies reagiert die Pulpa mit einer Entzündungsreaktion, die bei erfolgreicher Therapie allerdings ausheilen kann. Eine restlose Entfernung von Bakterien und bakterieller Produkte aus einer Kavität ist scheinbar nicht zwingend nötig, um eine solche Ausheilung zu erreichen ${ }^{6}$. Wichtig für das Pulpaüberleben scheint hingegen das Belassen einer Restdentinschichtdicke zwischen Läsion und Pulpa von mindestens $0,5 \mathrm{~mm}$ zu sein ${ }^{7,8}$. Der wichtigste Faktor ist, die Pulpa nicht zu eröffnen.

4. Im Rahmen der traditionellen „vollständigen“ Exkavation kann es vor allem zentral zu einer Überexkavation kommen, was die Gefahr der Pulpaexposition und die Wahrscheinlichkeit einer Diffusion zelltoxischer Materialien (Monomere) in die Pulpa erhöht ${ }^{9}$. Demnach sollte in tiefen Läsionen eine sogenannte selektive Exkavation vorgenommen werden. Dabei sind die Randbereiche der Kavität weiterhin vollständig zu exkavieren. In Pulpanähe kann hingegen auch Dentin, dass eine ledrige Konsistenz aufweist, belassen werden, wenn dies die Restdentinschichtdicke erhöht und eine Pulpaexpositionen verhindert ${ }^{10}$. Anschließend sollte eine dichte adhäsive Restauration platziert werden. Die zentral belassenen Bakterien werden so von der Kohlenhydratzufuhr abgeschnitten. Die Sklerosierung der Dentintubuli unter dem kariösen Dentin trägt weiterhin zu einer Inaktivierung der Bakterien bei. Die selektive Kariesexkavation gilt daher für tiefe, pulpanahe Läsionen als Therapie der Wahl. Der Patient sollte über die Therapie aufgeklärt werden, und es sollte ein Aktenvermerk angelegt werden, damit die röntgenologische Transluzenz unter der Restauration nicht als Sekundärkaries fehlbeurteilt wird. 
Bitte beantworten Sie erneut die unten aufgeführten Fragen, hat Sich an Ihrer Einstellung etwas geändert?

1. Welches Exkavationskriterium nutzen Sie und wann stoppen Sie die Exkavation im pulpanahen Dentindrittel bei einem vitalen Zahn, der keine Anzeichen einer irreversiblen Pulpitis aufweist? (Bitte in jeder Spalte einmal ankreuzen)

\begin{tabular}{|c|c|c|c|c|c|}
\hline \multicolumn{2}{|c|}{ Härte } & \multicolumn{2}{|c|}{ Farbe } & \multicolumn{2}{|c|}{ Feuchtigkeit } \\
\hline $\begin{array}{l}\text { Weiches } \\
\text { Dentin }\end{array}$ & $\square$ & $\begin{array}{l}\text { Stark } \\
\text { verfärbtes } \\
\text { Dentin }\end{array}$ & $\square$ & $\begin{array}{l}\text { Der } \\
\text { Kavitätenboden } \\
\text { ist stark feucht }\end{array}$ & $\square$ \\
\hline $\begin{array}{l}\text { Festes, noch } \\
\text { spanendes } \\
\text { Dentin }\end{array}$ & $\square$ & $\begin{array}{l}\text { Leicht } \\
\text { verfärbtes } \\
\text { Dentin }\end{array}$ & $\square$ & $\begin{array}{l}\text { Der } \\
\text { Kavitätenboden } \\
\text { ist leicht feucht }\end{array}$ & $\square$ \\
\hline $\begin{array}{l}\text { Hartes } \\
\text { Dentin } \\
\text { (Sondentest) }\end{array}$ & $\square$ & $\begin{array}{l}\text { Unverfärbtes } \\
\text { Dentin }\end{array}$ & $\square$ & $\begin{array}{l}\text { Der } \\
\text { Kavitätenboden } \\
\text { ist trocken }\end{array}$ & $\square$ \\
\hline $\begin{array}{l}\text { Die Härte ist } \\
\text { nicht wichtig }\end{array}$ & $\square$ & $\begin{array}{l}\text { Die Farbe ist } \\
\text { nicht wichtig }\end{array}$ & $\square$ & $\begin{array}{l}\text { Die } \\
\text { Feuchtigkeit ist } \\
\text { nicht wichtig }\end{array}$ & $\square$ \\
\hline
\end{tabular}

Wie sehr stimmen sie den folgenden Aussagen nun zu:

2. Bei einem vitalen Zahn, der keine Anzeichen einer irreversiblen Pulpitis aufweist, exkaviere ich pulpanah zurückhaltender als in der Kavitätenperipherie.

\begin{tabular}{|c|c|c|c|c|}
\hline Stimme zu & Stimme eher zu & $\begin{array}{c}\text { Stimme } \\
\text { teilweise zu }\end{array}$ & $\begin{array}{c}\text { Stimme eher } \\
\text { nicht zu }\end{array}$ & Stimme nicht zu \\
\hline$\square$ & $\square$ & $\square$ & $\square$ \\
\hline
\end{tabular}


3. Kariesbakterien sollten vollständig - auch in Pulpanähe - entfernt werden, da die Karies sonst voranschreitet.

\begin{tabular}{|c|c|c|c|c|}
\hline Stimme zu & Stimme eher zu & $\begin{array}{c}\text { Stimme } \\
\text { teilweise zu }\end{array}$ & $\begin{array}{c}\text { Stimme eher } \\
\text { nicht zu }\end{array}$ & Stimme nicht zu \\
\hline$\square$ & $\square$ & $\square$ & $\square$ & $\square$ \\
\hline
\end{tabular}

4. Bestimmte Mengen von kariösen Bakterien können belassen werden, da sie unter intakten Restaurationen versiegelt und inaktiviert werden

\begin{tabular}{|c|c|c|c|c|}
\hline Stimme zu & Stimme eher zu & $\begin{array}{c}\text { Stimme } \\
\text { teilweise zu }\end{array}$ & $\begin{array}{c}\text { Stimme eher } \\
\text { nicht zu }\end{array}$ & Stimme nicht zu \\
\hline
\end{tabular}

5. Wissenschaftliche Stellungnahmen und Empfehlungen haben einen Einfluss auf meine Behandlung.

\begin{tabular}{|c|c|c|c|c|}
\hline Stimme zu & Stimme eher zu & $\begin{array}{c}\text { Stimme } \\
\text { teilweise zu }\end{array}$ & $\begin{array}{c}\text { Stimme eher } \\
\text { nicht zu }\end{array}$ & Stimme nicht zu \\
\hline$\square$ & $\square$ & $\square$ & $\square$ & $\square$ \\
\hline
\end{tabular}

6. Ich exkaviere immer, auch pulpanah, bis ins harte Dentin, weil ich andere Dentinqualitäten nicht zuverlässig überprüfen kann.

\begin{tabular}{|c|c|c|c|c|}
\hline Stimme zu & Stimme eher zu & $\begin{array}{c}\text { Stimme } \\
\text { teilweise zu }\end{array}$ & $\begin{array}{c}\text { Stimme eher } \\
\text { nicht zu }\end{array}$ & Stimme nicht zu \\
\hline$\square$ & $\square$ & $\square$ & $\square$ & $\square$ \\
\hline
\end{tabular}




\begin{tabular}{|l|c|}
\hline Demographische Angaben \\
\hline Männlich \\
\hline Weiblich \\
\hline Alter \\
\hline Praktizierend seit \\
\hline Einzelpraxis \\
\hline Doppelpraxis \\
\hline Mehrbehandlerpraxis \\
\hline Universitätsklinik \\
\hline
\end{tabular}

\begin{tabular}{|l|c|}
\hline $\begin{array}{l}\text { Behandlungsschwerpunkt } \\
\text { (Mehrfachnennung möglich) }\end{array}$ \\
\hline Ästhetische ZHK & $\square$ \\
\hline Alterszahnheilkunde & $\square$ \\
\hline Endodontie & $\square$ \\
\hline Kinderzahnheilkunde & $\square$ \\
\hline Oralchirurgie & $\square$ \\
\hline Präventive ZHK & $\square$ \\
\hline Prothetische ZHK & $\square$ \\
\hline Restaurative ZHK & $\square$ \\
\hline
\end{tabular}

\title{
Vielen Dank für Ihre Teilnahme.
}

\author{
Quellenverzeichnis
}

1. Buchalla, W. et al. Aktuelle Empfehlungen zur Kariesexkavation. 72, 484-494 (2017).

2. Schwendicke, F. Selektive Kariesentfernung : Evidenz und Praxis - Chancen und Hürden. (2014).

3. Mertz-Fairhurst, E. J., Schuster, G. S., Williams, E. J. \& Fairhurst, C. W. Clinical progress of sealed and unsealed caries. Part II: Standardized radiographs and clinical observations. (1979).

4. Mertz-Fairhurst, E. J., Curtis, J. W. ., Ergle, J. W., Rueggenberg, F. A. \& Adair, S. M. Ultraconservative and cariostatic sealed restorations: results at year 10. J. Am. Dent. Assoc. 129, 55-66 (2001).

5. Kidd, E. A. M. How 'Clean' Must a Cavity Be before Restoration ? Caries Resaerch 38, 305-313 (2004).

6. Farges, J. et al. Odontoblast control of dental pulp inflammation triggered by cariogenic bacteria. 4, 1-3 (2013).

7. Smith, A. J. et al. Dentine as a bioactive extracellular matrix. Arch. Oral Biol. 57, 109-121 (2011).

8. Galler, K. M. et al. Influence of Root Canal Disinfectants on Growth Factor Release from Dentin. 41, 363-368 (2015).

9. Krifka, S., Spagnuolo, G., Schmalz, G. \& Schweikl, H. A review of adaptive mechanisms in cell responses towards oxidative stress caused by dental resin monomers. Biomaterials 34, 4555-4563 (2013).

10. Schwendicke, F. et al. Managing Carious Lesions: Consensus Recommendations on Carious Tissue Removal. Adv. Dent. Res. 28, 58-67 (2016). 


\section{CHARItÉ}

\section{CharitéCentrum für Zahn-, Mund- und Kieferheilkunde}

Charité | Campus Benjamin Franklin | 12200 Berlin

\author{
CharitéCentrum 3 für Zahn-, Mund-, und Kieferheilkunde \\ Charité - Universitätsmedizin Berlin \\ Abteilung für Zahnerhaltung und Präventivzahnmedizin \\ Direktor: Prof. Dr. Sebastian Paris \\ Standort: Assmannshauser-Str. 4-6 | 14197 Berlin \\ Korrespondenz: ZÄ Linda-Maria Jeggle \\ Tel. 030/ 450562358 \\ Fax 030/450 7562332 \\ Linda-Maria.Jeggle@charite.de \\ https://zahnerhaltung.charite.de/
}

Berlin, 05. März 2018

\section{Umfrage für Zahnärztinnen und Zahnärzte zur Exkavation tiefer kariöser Läsionen}

Liebe Kollegin, lieber Kollege,

in der vorliegenden Umfrage untersuchen wir, wie deutsche Zahnärztinnen und Zahnärzte tiefe kariöse Läsionen in vitalen Zähnen behandeln. Wir würden uns freuen, wenn Sie sich ein paar Minuten Zeit nehmen und den vorliegenden Fragebogen ausfüllen könnten.

Der Fragebogen enthält 12 Fragen. Die Beantwortung wird etwa 10 Minuten dauern. Wir bitten Sie, alle Fragen sorgfältig zu lesen und zu beantworten. Es gibt weder richtige noch falsche Antworten. Der erste Teil des Bogens erfragt Ihre jetzige Einstellung und Ihr Verhalten bei der Therapie der geschilderten Kariesläsionen. Im zweiten Teil untersuchen wir, ob Sie dieses Verhalten unter bestimmten Umständen ändern würden, dafür liegt ein gesonderter Umschlag bei. Bitte öffnen Sie diesen erst nach Bearbeitung des ersten Teils. Er enthält einen kurzen Text und den zweiten Teil der Fragen. Nach Beantwortung aller 12 Fragen können Sie alle Seiten in den beigelegten Rückumschlag geben und kostenfrei an uns zurücksenden. Die erhobenen Daten werden anonymisiert evaluiert und nach datenschutzrechtlichen Vorschriften verarbeitet.

Wir bedanken uns für Ihre Mühe im Voraus. 
1. Welches Exkavationskriterium nutzen Sie und wann stoppen Sie die Exkavation im pulpanahen Dentindrittel bei einem vitalen Zahn, der keine Anzeichen einer irreversiblen Pulpitis aufweist? (Bitte in jeder Spalte einmal ankreuzen)

\begin{tabular}{|l|l|l|l|l|}
\hline \multicolumn{1}{|c|}{ Härte } & \multicolumn{1}{|c|}{ Farbe } & \multicolumn{1}{|c|}{ Feuchtigkeit } \\
\hline $\begin{array}{l}\text { Weiches } \\
\text { Dentin }\end{array}$ & $\square$ & $\begin{array}{l}\text { Stark } \\
\text { verfärbtes } \\
\text { Dentin }\end{array}$ & $\square$ & $\begin{array}{l}\text { Der } \\
\text { Kavitätenboden } \\
\text { ist stark feucht }\end{array}$ \\
\hline $\begin{array}{l}\text { Festes, noch } \\
\text { spanendes } \\
\text { Dentin }\end{array}$ & $\square$ & $\begin{array}{l}\text { Leicht } \\
\text { verfärbtes } \\
\text { Dentin }\end{array}$ & $\square$ & $\begin{array}{l}\text { Der } \\
\text { Kavitätenboden } \\
\text { ist leicht feucht }\end{array}$ \\
\hline $\begin{array}{l}\text { Hartes } \\
\text { Dentin } \\
\text { (Sondentest) }\end{array}$ & $\square$ & $\begin{array}{l}\text { Unverfärbtes } \\
\text { Dentin }\end{array}$ & $\square$ & $\begin{array}{l}\text { Der } \\
\text { Kavitätenboden } \\
\text { ist trocken }\end{array}$ \\
\hline $\begin{array}{l}\text { Die Härte ist } \\
\text { nicht wichtig }\end{array}$ & $\square$ & $\begin{array}{l}\text { Die Farbe ist } \\
\text { nicht wichtig }\end{array}$ & $\square$ & $\begin{array}{l}\text { Die } \\
\text { Feuchtigkeit ist }\end{array}$ \\
\hline
\end{tabular}

Wie sehr stimmen sie den folgenden Aussagen zu:

2. Bei einem vitalen Zahn, der keine Anzeichen einer irreversiblen Pulpitis aufweist, exkaviere ich pulpanah zurückhaltender als in der Kavitätenperipherie.

\begin{tabular}{|c|c|c|c|c|}
\hline Stimme zu & Stimme eher zu & $\begin{array}{c}\text { Stimme } \\
\text { teilweise zu }\end{array}$ & $\begin{array}{c}\text { Stimme eher } \\
\text { nicht zu }\end{array}$ & Stimme nicht zu \\
\hline$\square$ & $\square$ & $\square$ & $\square$ & $\square$ \\
\hline
\end{tabular}

3. Kariesbakterien sollten vollständig - auch in Pulpanähe - entfernt werden, da die Karies sonst voranschreitet.

\begin{tabular}{|c|c|c|c|c|}
\hline Stimme zu & Stimme eher zu & $\begin{array}{c}\text { Stimme } \\
\text { teilweise zu }\end{array}$ & $\begin{array}{c}\text { Stimme eher } \\
\text { nicht zu }\end{array}$ & Stimme nicht zu \\
\hline$\square$ & $\square$ & $\square$ & $\square$ & $\square$ \\
\hline
\end{tabular}


4. Bestimmte Mengen von kariösen Bakterien können belassen werden, da sie unter intakten Restaurationen versiegelt und inaktiviert werden.

\begin{tabular}{|c|c|c|c|c|}
\hline Stimme zu & Stimme eher zu & $\begin{array}{c}\text { Stimme } \\
\text { teilweise } z u\end{array}$ & $\begin{array}{c}\text { Stimme eher } \\
\text { nicht zu }\end{array}$ & Stimme nicht zu \\
\hline$\square$ & $\square$ & $\square$ & $\square$ & $\square$ \\
\hline
\end{tabular}

5. Wissenschaftliche Stellungnahmen und Empfehlungen haben einen Einfluss auf meine Behandlung.

\begin{tabular}{|c|c|c|c|c|}
\hline Stimme zu & Stimme eher zu & $\begin{array}{c}\text { Stimme } \\
\text { teilweise zu }\end{array}$ & $\begin{array}{c}\text { Stimme eher } \\
\text { nicht zu }\end{array}$ & Stimme nicht zu \\
\hline
\end{tabular}

6. Ich exkaviere immer, auch pulpanah, bis ins harte Dentin, weil ich andere Dentinqualitäten nicht zuverlässig überprüfen kann.

\begin{tabular}{|c|c|c|c|c|}
\hline Stimme zu & Stimme eher zu & $\begin{array}{c}\text { Stimme } \\
\text { teilweise zu }\end{array}$ & $\begin{array}{c}\text { Stimme eher } \\
\text { nicht zu }\end{array}$ & Stimme nicht zu \\
\hline$\square$ & $\square$ & $\square$ & $\square$ & $\square$ \\
\hline
\end{tabular}

Bitte öffnen Sie nun den Umschlag mit dem zweiten Teil der Befragung, und beantworten Sie die enthaltenen Fragen nach dem Lesen eines kurzen Textes. 


\section{Kariesexkavator $\mathbf{2 . 0}$}

Für die Exkavation von tiefen kariösen Läsionen gibt es verschiedene Exkavationsstrategien. Neben der traditionellen „vollständigen“ Exkavation wird mittlerweile auch eine sogenannte selektive Exkavation propagiert. Dabei soll in Pulpanähe schonender exkaviert werden. Stellen Sie sich vor, es würde ein Hilfsmittel entwickelt werden, um Ihnen die selektive Exkavation zu erleichtern:

Stellen Sie sich beispielsweise vor, es gibt ein neues Winkelstück mit einer integrierten Funktion zur Unterstützung der selektiven Kariesexkavation bei tiefen Läsionen. Das neue Winkelstück ersetzt das grüne Winkelstück bei der Exkavation, arbeitet aber weiter mit Rosenbohrern. So lange Sie in der Peripherie arbeiten, wird wie gewohnt exkaviert. Sobald Sie in pulpanahe Areale vordringen, müssen Sie an dem Winkelstück einen Umschaltknopf drücken. Das Winkelstück hat präzise Detektoren, die nun im selektiven Exkavationsmodus die Härte des Dentins und den Anpressdruck messen. Weiterhin misst es mittels einer integrierten Kamera die Fluoreszenzeigenschaften des Dentins. Das Winkelstück zeigt Ihnen an, wann Sie ausreichend selektiv exkaviert haben, verhindert gleichzeitig eine Überexkavation. Die Kosten für dieses Winkelstück sind nur geringfügig höher als die für ein herkömmliches grünes Winkelstück.

Bitte nehmen Sie an, Sie hätten die Möglichkeit ein solches Instrument zu kaufen, und beantworten Sie erneut die Fragen unten.

Bitte beantworten Sie erneut die unten aufgeführten Fragen, hat Sich an Ihrer Einstellung etwas geändert? 
1. Welches Exkavationskriterium nutzen Sie und wann stoppen Sie die Exkavation im pulpanahen Dentindrittel bei einem vitalen Zahn, der keine Anzeichen einer irreversiblen Pulpitis aufweist? (Bitte in jeder Spalte einmal ankreuzen)

\begin{tabular}{|l|l|l|l|l|}
\hline \multicolumn{1}{|c|}{ Härte } & \multicolumn{1}{|c|}{ Farbe } & \multicolumn{1}{|c|}{ Feuchtigkeit } \\
\hline $\begin{array}{l}\text { Weiches } \\
\text { Dentin }\end{array}$ & $\square$ & $\begin{array}{l}\text { Stark } \\
\text { verfärbtes } \\
\text { Dentin }\end{array}$ & $\square$ & $\begin{array}{l}\text { Der } \\
\text { Kavitätenboden } \\
\text { ist stark feucht }\end{array}$ \\
\hline $\begin{array}{l}\text { Festes, noch } \\
\text { spanendes } \\
\text { Dentin }\end{array}$ & $\square$ & $\begin{array}{l}\text { Leicht } \\
\text { verfärbtes } \\
\text { Dentin }\end{array}$ & $\square$ & $\begin{array}{l}\text { Der } \\
\text { Kavitätenboden } \\
\text { ist leicht feucht }\end{array}$ \\
\hline $\begin{array}{l}\text { Hartes } \\
\text { Dentin } \\
\text { (Sondentest) }\end{array}$ & $\square$ & $\begin{array}{l}\text { Unverfärbtes } \\
\text { Dentin }\end{array}$ & $\square$ & $\begin{array}{l}\text { Der } \\
\text { Kavitätenboden } \\
\text { ist trocken }\end{array}$ \\
\hline $\begin{array}{l}\text { Die Härte ist } \\
\text { nicht wichtig }\end{array}$ & $\square$ & $\begin{array}{l}\text { Die Farbe ist } \\
\text { nicht wichtig }\end{array}$ & $\square$ & $\begin{array}{l}\text { Die } \\
\text { Feuchtigkeit ist }\end{array}$ \\
\hline
\end{tabular}

Wie sehr stimmen sie den folgenden Aussagen nun zu:

2. Bei einem vitalen Zahn, der keine Anzeichen einer irreversiblen Pulpitis aufweist, exkaviere ich pulpanah zurückhaltender als in der Kavitätenperipherie.

\begin{tabular}{|c|c|c|c|c|}
\hline Stimme zu & Stimme eher zu & $\begin{array}{c}\text { Stimme } \\
\text { teilweise zu }\end{array}$ & $\begin{array}{c}\text { Stimme eher } \\
\text { nicht zu }\end{array}$ & Stimme nicht zu \\
\hline$\square$ & $\square$ & $\square$ & $\square$ & $\square$ \\
\hline
\end{tabular}


3. Kariesbakterien sollten vollständig - auch in Pulpanähe - entfernt werden, da die Karies sonst voranschreitet.

\begin{tabular}{|c|c|c|c|c|}
\hline Stimme zu & Stimme eher zu & $\begin{array}{c}\text { Stimme } \\
\text { teilweise zu }\end{array}$ & $\begin{array}{c}\text { Stimme eher } \\
\text { nicht zu }\end{array}$ & Stimme nicht zu \\
\hline$\square$ & $\square$ & $\square$ & $\square$ & $\square$ \\
\hline
\end{tabular}

4. Bestimmte Mengen von kariösen Bakterien können belassen werden, da sie unter intakten Restaurationen versiegelt und inaktiviert werden

\begin{tabular}{|c|c|c|c|}
\hline Stimme zu & Stimme eher zu & $\begin{array}{c}\text { Stimme } \\
\text { teilweise zu }\end{array}$ & $\begin{array}{c}\text { Stimme eher } \\
\text { nicht zu }\end{array}$ \\
\hline$\square$ & $\square$ & $\square$ & Stimme nicht zu \\
\hline
\end{tabular}

5. Wissenschaftliche Stellungnahmen und Empfehlungen haben einen Einfluss auf meine Behandlung.

\begin{tabular}{|c|c|c|c|c|}
\hline Stimme zu & Stimme eher zu & $\begin{array}{l}\text { Stimme } \\
\text { teilweise zu }\end{array}$ & $\begin{array}{l}\text { Stimme eher } \\
\text { nicht zu }\end{array}$ & Stimme nicht zu \\
\hline$\square$ & $\square$ & $\square$ & $\square$ & $\square$ \\
\hline
\end{tabular}

6. Jetzt, wo Sie theoretisch ein Werkzeug zur Überprüfung der Dentinqualitäten zur Verfügung haben, würden Sie bevorzugt selektiv exkavieren?

\begin{tabular}{|c|c|c|c|c|}
\hline Stimme zu & Stimme eher zu & $\begin{array}{c}\text { Stimme } \\
\text { teilweise } z u\end{array}$ & $\begin{array}{l}\text { Stimme eher } \\
\text { nicht zu }\end{array}$ & Stimme nicht zu \\
\hline$\square$ & $\square$ & $\square$ & $\square$ & $\square$ \\
\hline
\end{tabular}




\begin{tabular}{|l|c|}
\hline Demographische Angaben & $\square$ \\
\hline Männlich & $\square$ \\
\hline Weiblich & \\
\hline Alter & \\
\hline Praktizierend seit & $\square$ \\
\hline Einzelpraxis & $\square$ \\
\hline Doppelpraxis & $\square$ \\
\hline Mehrbehandlerpraxis & \\
\hline Universitätsklinik & \\
\hline
\end{tabular}

\begin{tabular}{|l|c|}
\hline $\begin{array}{l}\text { Behandlungsschwerpunkt } \\
\text { (Mehrfachnennung möglich) }\end{array}$ & $\square$ \\
\hline Ästhetische ZHK & $\square$ \\
\hline Alterszahnheilkunde & $\square$ \\
\hline Endodontie & $\square$ \\
\hline Kinderzahnheilkunde & $\square$ \\
\hline Oralchirurgie & $\square$ \\
\hline Präventive ZHK & \\
\hline Prothetische ZHK & $\square$ \\
\hline Restaurative ZHK & $\square$ \\
\hline
\end{tabular}

Vielen Dank für Ihre Teilnahme. 


\section{Lebenslauf}

Mein Lebenslauf wird aus datenschutzrechtlichen Gründen in der elektronischen Version meiner Arbeit nicht veröffentlicht. 


\section{Publikationsliste}

1. Jeggle L, Baker S, Schwendicke F

Changing Dentists' Carious Tissue Removal Behavior: Qualitative Study and Behavioral Change Simulation Experiment. J Dent 2018;81(2019):43-51. Impact Factor: 3,770 


\section{Danksagung}

Mein herzlicher Dank gilt meinem Doktorvater PD Dr. Schwendicke für die Überlassung des Themas, die tolle Betreuung und Unterstützung sowie Ermutigung und Förderung zu jedem Zeitpunkt dieses Projektes.

Herrn Professor Dr. Paris gilt mein Dank für die Möglichkeit wissenschaftlich zu arbeiten, diese Arbeit in seiner Abteilung fertigen zu dürfen und mich dabei beruflich weiterzuentwickeln.

Ich danke im Besonderen meiner Familie: Meinen Eltern Karina und Christoph, meinen Geschwistern Jenny und Julian sowie meiner Oma Annemarie und meiner Cousine Esther. Danke, dass ihr mich unermüdlich unterstützt und motiviert habt.

Danke an Dich Christian, dass Du immer an mich geglaubt hast und an meiner Seite bist, seit dem ersten Tag meines Studiums. 